\title{
L'HOMME L'Homme
}

165 | janvier-mars 2003

Image et anthropologie

\section{Le masque de la raie}

Étude ethno-astronomique de l'iconographie d'un masque rituel miraña

\section{Dimitri Karadimas}

\section{(2) OpenEdition}

Journals

Édition électronique

URL : http://journals.openedition.org//homme/202

DOI : $10.4000 /$ Ihomme.202

ISSN : 1953-8103

Éditeur

Éditions de l'EHESS

Édition imprimée

Date de publication : 1 janvier 2003

Pagination : 173-204

ISBN : 2-7132-1779-2

ISSN : 0439-4216

Référence électronique

Dimitri Karadimas, « Le masque de la raie », L'Homme [En ligne], 165 | janvier-mars 2003, mis en ligne le 27 mars 2008, consulté le 01 mai 2019. URL : http://journals.openedition.org/lhomme/202 ; DOI :

10.4000/lhomme.202 


\title{
Le masque de la raie Étude ethno-astronomique de l'iconographie d'un masque rituel miraña
}

\author{
Dimitri Karadimas
}

P LUSIEURS des sociétés indigènes du Nord-Ouest amazonien - Miraña, Bora, Uitoto, Andoques (langues isolées), Yukuna, Matapi (Arawak), Tanimuka, Letuama, et Makuna (Tukano orientaux) - possèdent en commun un rituel à l'occasion duquel sont bues d'importantes quantités de bière brassée à partir de la pulpe macérée des fruits du palmier parépou (Bactris gasipaes, aussi connu comme pupunha au Brésil, chontaduro en Colombie et au Pérou, et pijuayo dans une autre partie du Pérou). Lors de ce rituel, les esprits des animaux sont invités à danser et à festoyer avec les humains. Ces " esprits" prennent la forme de masques et de costumes que revêt une partie des invités et des amphitryons, formant en alternance un groupe de danseurs qui occupe l'intérieur de la maloca (maison communautaire), alors que l'autre, au dehors, prépare et répète le chant de sa prochaine entrée. Une multitude d'animaux ainsi que plusieurs êtres imaginaires - incarnés chacun par une danse et un chant, plus d'ailleurs que par un masque spécifique -, défilent ainsi durant une journée et une nuit dans un ballet ininterrompu.

Sur les cent soixante-dix espèces (en moyenne) qui entrent dans la maloca pour effectuer une danse, chanter et recevoir en échange de la part des humains de la bière de parépou, seuls quelques masques représentent une espèce en particulier (certains scarabées, une espèce de guêpe, les abeilles, le fourmilier, le jaguar et l'ocelot, le grand hocco [Crax alector], la libellule, le papillon morpho, la

Ce texte, rédigé en décembre 1999, fait suite au colloque "Art indigène et anthropologie " organisé par la Société suisse des américanistes à Trujillo (Espagne) en octobre de la même année ; les principales idées ont été présentées au séminaire Cartographie de l'immatériel, en avril 2000 à Paris XNanterre, et à l'Université de St. Andrews en Écosse en mai 2000. Je tiens à remercier vivement Claude Lévi-Strauss pour sa lecture critique du manuscrit et les nouvelles pistes de recherches qu'il a bien voulu me suggérer. Je suis aussi reconnaissant aux différents organisateurs du colloque et des séminaires dont Gerhard Baer et Manuel Gutierrez-Estevez, Alain Breton et Marie-Pierre Bousquet, Tristan Platt et Joanna Overing. J'exprime ma gratitude à France-Marie Renard-Casevitz pour ses conseils lors de l'élaboration de ce travail, ainsi qu’à Eduardo Viveiros de Castro, Emmanuel Désveaux, Nicolas Journet et Jean-Pierre Chaumeil pour les remarques et commentaires qu'ils ont bien voulu apporter à ce texte, et à Philippe Blanchot pour les photographies des masques en studio. 
chauve-souris et quelques autres). Le reste des espèces est représenté par deux masques; l'un qui incarne les espèces aquatiques, et l'autre celles des animaux terrestres et aériens ${ }^{1}$.

C'est plus particulièrement à l'étude de l'iconographie des motifs du premier type de masque (espèces du milieu aquatique) suivant une interprétation d'une version d'un mythe miraña que je m'attacherai. Selon les intéressés, le rituel commémore l'épisode mythique de l'origine du premier parépou, volé au peuple des poissons par le héros culturel pour le rapporter sur terre. Mon propos est de montrer comment ces motifs et, par delà, ce type de masque, évoquent une particularité astronomique liée aux équinoxes; le rituel a lieu autour de celui de mars alors que les fruits du palmier parépou mûrissent deux fois dans l'année lors des équinoxes (mars et septembre). Il s'agira également de montrer comment les motifs de ces masques ne constituent nullement de simples représentations abstraites de visages - d'êtres humains ou de déités -, mais plutôt, une mise à profit d'une identité de structures entre l'architecture des lignes du visage et certaines particularités astronomiques perçues à ces dates - et dessinées sur les masques.

Pour ce faire, je ferai une description rapide de l'aire de répartition du rituel et de son déroulement pour ensuite aborder le mythe. J'en proposerai une interprétation astronomique afin de montrer comment ce mythème a été rendu en tant que motif sur les masques et d'expliquer la variation des formes de ces derniers. Mon propos n'est pas d'expliciter ni d'interpréter le rituel en lui-même, étant donné qu'une quantité importante d'autres masques participe aux festivités. Cependant, aussi bien l'assise mythique que les implications astronomiques qu'elle induit me paraissent essentielles pour qui veut se lancer dans linterprétation de ce rituel pour l'ensemble des sociétés qui le pratiquent dans la région ${ }^{2}$.

Il est a noter que les études comparatives des masques amazoniens sont relativement peu fournies au regard de celles des autres continents : un article récent de G. Baer (1993) fait état de la question : ceux traitant de l'iconographie sont encore plus rares (cf. Zerries 1961, 1981; Hartmann 1967, Münzel 1971, Vincent 1986, Erikson 1996, Goulard 2000-2001).

Cet article se place dans la continuation d'un travail (Karadimas 1999) dans lequel je présente une interprétation astronomique de la première partie de la version du mythe en association avec plusieurs objets archéologiques en provenance des Andes colombiennes. J'étudierai ici la seconde partie consacrée à l'acquisition du premier palmier parépou par le héros culturel. Je ne rappellerai que les conclusions les plus importantes auxquelles je suis parvenu, notamment sur les aspects astronomiques, en demandant au lecteur de se reporter à mon article afin d'obtenir les démonstrations nécessaires à leur validation.

1. Plusieurs masques en provenance de cette région ont été collectés dans les années 70 (collections Jacopin, Gasché, Gu yot) et se tro u vent au Musée d'ethnographie de Neuchâtel (cf. catalogue de l' exposition, Amazonie du Nord-Ouest (Musée d'ethnographie 1975).

2. Il n'existe, à ma connaissance, qu'une interprétation de ce rituel réalisée par K. Århem pour les Makuna de l'Apaporis (1998); et, il y a quelques années, J. Gasché a réalisé un film sur le déroulement d'une fête du même type chez les Bora de l'Igara-Paraná en Colombie. 


\section{La fête de la bière de parépou}

La fête de mémébà (litt. "jus des fruits de parépou ») consiste essentiellement à inviter les esprits des animaux à venir boire de la bière et à festoyer avec les humains une fois l'an, au moment de la grande période de fructification de ce palmier, autour de l'équinoxe de mars. Durant l'année miraña qui débute alors, les humains attendent en contrepartie que les animaux les reçoivent en forêt aussi bien qu'ils ont été reçus. L'échange et la rencontre portent sur la nourriture de chacun; les uns se nourrissent des "fruits" des "maitres des animaux " (le gibier), alors que les autres viennent boire des fruits des humains (Bactris gasipaes est le seul palmier amazonien réellement anthropique qui ne pousse pas à l'état sauvage) avec, toutefois, une différence; dans les temps mythiques, ce palmier appartenait exclusivement au peuple des poissons dans le monde souterrain. Le rituel commémore en fait un épisode mythique : le vol par le héros culturel du premier noyau de parépou rapporté sur la terre ferme, la guerre avec les poissons, et la victoire finale du héros sur son rival Raie.

Il existe différentes variantes de ce rituel suivant les sociétés de la région qui l'organisent. Les groupes évoqués sont tous voisins et, même s'ils appartiennent

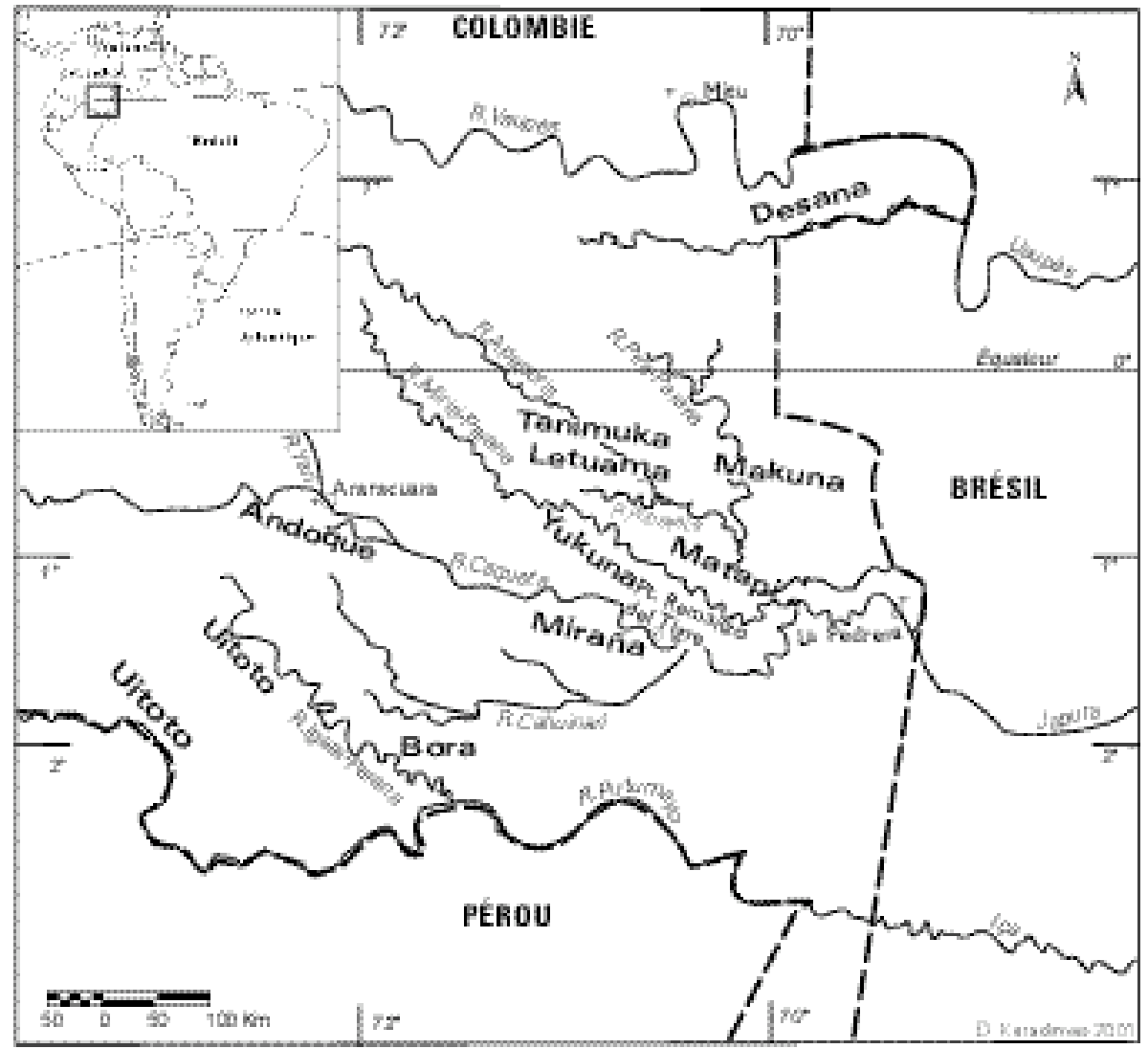

Localisation des groupes cités 
à des groupes linguistiques différents, ils partagent plusieurs aspects culturels, mythologiques et rituels. À telle enseigne que les deux rituels des esprits des animaux auxquels j'ai pu participer (en mars 1992 et en mai 1993) étaient des invitations mutuelles entre Yukuna et Miraña du fleuve Caquetá. Les intéressés s'accordent pour reconnaître que, sous sa forme actuelle, c'est-à-dire avec les entités et les masques qu'ils sont censés incarner, l'origine de ce rituel doit être attribuée soit aux Tanimuka (pour les Miraña), soit aux Letuama (pour les Makuna, cf. Århem 1998: 142); en tout cas, il semble que l'épicentre de son origine doive être reconnu aux groupes situés en Colombie, entre l'Apaporis et le MiritiParaná. Cependant, les Miraña affirment que ce rituel existe chez eux sous une forme légèrement différente, et que c'est à l'ensemble de ces groupes que les "esprits" ont confié les chants dans un temps mythique. Ainsi, les Bora, les Andoque et les Uitoto possèdent des formes spécifiques d'exécution du rituel, et T. Whiffen (1915) avait déjà assisté à ce type de festivité au début de ce siècle. Enfin, la version mythique qui accrédite pour les Miraña le déroulement du rituel fait partie de la longue geste de "Souffleur de Sarbacane » - héros culturel et personnage clé de la mythologie miraña.

Les Miraña font précéder - ou parfois suivre - la partie du rituel dédiée à l'invitation et à la danse des esprits des animaux par " la danse des poissons ", qui est entièrement dédiée à la commémoration du peuple de l'eau, durant laquelle aucune bière de parépou n'est bue et où les participants ne portent ni costumes ni masques. La seule composante de travestissement présente dans cette première phase rituelle est une palme portée horizontalement sur l'épaule, dont la nervure centrale (ou rachis) et les pennes (ou folioles) représentent respectivement l'arête centrale et les arrêtes latérales qui en émanent. Les danseurs n'utilisent que des hochets comme instruments de musique pendant cette première phase, alors que des grelots amarrés à la cheville servent d'instrument principal lors de la fête de la bière qui suit (pour certains chants, les grelots sont associés avec des flûtes de pan et à d'autres instruments).

Dans tous les cas de figure, le rituel se termine aujourd'hui par un tournoi de football, qui est venu remplacer l'ancien jeu de balle miraña (joué avec une balle de caoutchouc frappée à la mode méso-américaine avec les hanches et surtout les genoux). Cette dernière phase fait partie intégrante du rituel et commémore la joute exécutée par le héros mythique chez le peuple des poissons. Lors de sa première visite dans le monde aquatique, les poissons - ses beaux-frères - jouaient à la balle de caoutchouc et invectivaient le héros en lui faisant re ma rquer " qu'il n'avait pas de genou» (= qu'il n'avait pas de courage ou, plus exactement, qu'il avait les genoux qui flanchent). Répondant à la provocation de ses beaux-frères, le héros, par un magistral tir du genou, décroche le racème de parépou qui tombe à terre : les fruits éclatent et laissent échapper leur noyau.

Le rituel n'est cependant pas exclusivement dédié au monde aquatique mais plutôt à l'ensemble des espèces " animales ». Il n'y a pas de présentation de végétaux ou de minéraux; il existe en revanche une entrée d'un masque appelé "celui qui répète ", incarnation de l'écho, ainsi que de plusieurs masques qui évoquent des 
états de la personne, des rencontres en forêt, comme des enfants ou personnes perdues, etc. Chacune de ces entités entre dans la maloca en effectuant un chant et des pas de danse qui miment son comportement. Elles se présentent alors au maître de maloca, reçoivent de la coca et du tabac et repartent danser (au début du rituel, les singes reçoivent de la nourriture au centre de la maloca : les danseurs - les esprits des animaux - mangent en dehors, et non avec les humains). Avant leur sortie par la porte principale, chacun des danseurs reçoit individuellement une calebasse remplie de bière de parépou qu'il doit boire sur le champ. Les masques ne sont pas dotés d'orifice à hauteur de bouche qui permettrait au danseur de l'absorber; ils sont donc obligés de soulever le masque pour boire. C'est à ce moment, affirment les Miraña, que l'on reconnaît réellement qui est venu danser, non pas dans le sens de l'identité de la personne qui porte le masque, mais de l'espèce en particulier. Ainsi, toujours selon les Miraña, c'est à ce moment que l'on peut voir sur le visage des danseurs les stigmates du caractère de l'espèce. Celui qui donne la calebasse de bière de parépou dit alors de quelle espèce il s'agit. Les Miraña affirment reconnaître la raie, le crabe, la loutre, le pécari, etc., dans l'expression du visage et dans le regard du danseur. Il s'agit donc bien de l'esprit (ou le caractère, qui s'exprime dans le chant et le pas de danse) de telle espèce qui donne vie au masque plutôt que l'inverse (ce n'est pas tant le masque qui donne le caractère au danseur puisqu'il n'y a, en tout et pour tout, qu'une quinzaine de masques différents alors qu'il y a dix fois plus d'espèces qui viennent visiter les humains). Il s'agit donc, comme tout masque, d'un artifice qui cache et montre en même temps, si ce n'est qu'il cache l'animalité possible d'un visage humain tout en montrant l'humanité possible d'un esprit (d'une face) animal(e). In versement, sans masque, l'humanité exprimée par les visages est, de ce fait, également un masque - la persona -, puisque ce visage peut prendre des traits animaux; l'humanité n'est donc, pour les Miraña, qu'un comportement, qu'une manière d'être qui s'exprime et peut être reconnue, au même titre que l'animalité, dans le regard et les traits de la face (qui est, ou non, grimaçante). La première est socialement acceptée, la seconde est voilée par un masque représentant une animalité "humaine». La mise en rapport du visage humain et animal passe ainsi par une "mise en masque" de la personne: les masques et les habits du rituel sont ainsi une tentative, de la part des esprits des animaux, de se montrer sous un jour humanisé, là où les humains se dotent $\mathrm{d}$ 'artefacts où de comportements animaux lorsqu'ils visitent les esprits pour négocier les animaux chassés. En ce sens, il s'agit d'une visite des esprits venu négocier des corps, ou une nourriture d'humains (d'un rouge soutenu, la bière de parépou est souvent comparée, par les Miraña, à du sang : elle renvoie tout au moins cette image aux esprits des animaux). Les prédations mutuelles entre ces deux sphères sont donc implicitement évoquées dans le rituel ; les morts sont aussi présents puisqu'ils se retro uvent inclus dans la catégorie des gens de l'ailleurs.

Ces masques possèdent toutefois des identités mythologiques particulières et ne peuvent être substitués les uns aux autres, tant il est vrai que le rituel suit une trame mythique. Ainsi, le masque qui représente les animaux terre st res et aériens est toujours doté de plumes, de poils, et d'un rictus laissant apparaître sa denti- 
tion. Ce masque incarne mé:ì, aussi désigné comme le "mâtre des animaux» (íámè nì:mútê) et de ces deux domaines (cf. Karadimas 1997). Ce maitre des animaux est identifié à certains personnages mythiques et porte plusieurs noms. L'un d'eux est le héros mythique "Souffleur de Sarbacane", voleur du premier noyau de parépou qui, en tant que fils d'Astre, reprend le nom de son défunt père (cf. infra). Dans le mythe, il est directement désigné par le peuple des poissons comme "Astre" ou "Soleil du milieu " (pínè núbà) (nous reviend rons sur ce nom pour savoir de quel «milieu " il s'agit); "Soleil de la Guer re» (t́mékò núbà) est l'autre nom qu'il porte pour avoir combattu les poissons et institué ainsi la pratique de la guerre et de l' a $\mathrm{n}$ th ropophagie (les poissons étaient alors des personnes, comme les humains sont aujourd'hui des "poissons " pour les esprits ; c'est-à-dire la nourriture la moins polluée qui, pour les Miraña, est à associer au végétal) ${ }^{3}$.

Les masques entrent souvent par paires et certains informateurs affirment que l'un est le maître des animaux et l'autre "Anaconda des poissons », le maître - ou le capitán - des espèces aquatiques. En contradiction avec les premiers, d'autres informateurs affirment que Anaconda des poissons est incarné par l'autre type de masque, dépourvu de ces ornements de plumes et de poils, masque qui représenterait successivement plusieurs entités : en premier lieu les espèces aquatiques et leur maître "Anaconda des poissons" (beau-père du héros culturel dans le mythe) ; mais aussi les ádjénè, terme qui fait référence aux grands-pères, aux anciens, ce qui inclut également les « ancêtres" (les esprits des morts), venus du ciel (territoire des étoiles). Lors de leur première entrée, les danseurs qui portent ces masques s'alignent près du maître de la fête. En même temps que de la coca et du tabac en poudre leur sont offerts, on leur demande d'où ils viennent. N'arrivant pas à parler et regardant la personne qui les questionne en bougeant la tête avec des mouvements interrogatifs, ils utilisent un gros cigare qu'on leur a préalablement tendu et qu'ils brandissent en décrivant un trajet zigzaguant de haut en bas. Dans la pénombre de la maloca, la partie incandescente du cigare représente une étoile et le mouvement décrit son trajet jusqu'aux humains. Il existe donc un impact astronomique dans les identités successives des personnages masqués. Ces mêmes masques sont aussi utilisés durant le rituel pour incarner d'autres espèces qui ne sont pas toutes d'origine aquatique.

À la fin du rituel enfin, une passe de danse est réservée aux adieux effectués par les danseurs. Dotés du masque du maitre des animaux, ils entrent tous par la porte principale en plaçant le deuxième masque sur l'épaule à la façon d'un baluchon. C'est le seul moment durant tout le rituel où deux masques sont portés ensembles par un danseur. Il faut toutefois noter que le danseur n'enfile qu'un seul des deux masques sur la tête; le second est mis en bandoulière sur l'épaule par les fibres noires qui représentent sa chevelure nouée en natte, avec le visage du masque apparent. Ce second masque est inanimé, on peut le considérer comme " mort»; il n'est que la peau d'un visage, portée là comme une tête trophée par le Maître des

3. Je renvoie le lecteur à ma thèse de doctorat (Karadimas 1997) pour une compréhension plus globale de la perception des humains, par les entités et les esprits, comme des végétaux-poissons. 
Animaux ou, selon le mythe, par Soleil du Milieu. D'ailleurs, les danseurs tentent de faire concorder cette phase avec le lever du soleil. C'est à ce moment que le rituel fait explicitement référence au mythe puisqu'il s'agit de représenter la victoire de Soleil du Milieu sur son rival Raie qu'il tue d'un coup de lance en plein cœur. À ce moment du rituel, ce masque devient l'incarnation de Raie, il est celui de la raie, victime de Soleil du Milieu. Pour les Yukuna, il existe un raccourci conceptuel entre les différentes valeurs à accorder à ce type de masque : la raie est le capitán de los peces, «le maître des poissons» (cf. Van der Hammen 1992 : 245, 263). Le masque qui représente le «maitre des poissons » chez les Miraña et les Yukuna est, chez les premiers, l'anaconda, alors qu'il est la raie chez les seconds. Les Andoque voient dans la raie la tête transformée d'un anaconda (cf. Landaburu \& Pineda 1984) et, comme les Uitoto, la classent dans la même catégorie (Bu rtch 1983: 65, 200). Nous verrons avec le mythe miraña que c'est au personnage Raie qu'il faut accorder la précédence quant à l'identité du masque. Les Miraña classent la raie parmi les anacondas: dire que le masque du Maître des eaux est un anaconda, c'est faire référence à la classe, dire que c'est une raie est en indiquer l'espèce.

Quoi qu'il en soit, les esprits des animaux font alors plusieurs fois le tour à l'intérieur, puis a l'extérieur de la maloca, et reprennent le chemin de la forêt. Un tir à blanc clôt aujourd'hui cette phase du rituel pour laisser place, dans l'après-midi du même jour, à la partie de football.

\section{Le mythe du premier palmier parépou}

Le résumé qui suit reprend les séquences essentielles d'un très long mythe - jamais raconté en une seule fois -, de la geste de "Souffleur de Sarbacane ». Le mythe débute dans le ciel avec le père du héros.

\section{Première partie}

«Astre» («du jour» pour le soleil, et «de la nuit» pour la lune), suite à son mariage avec une femme dénommée Kinkajou (Potos flavus) se tro uve confronté à ses beauxfrères douroucoulis (Aotus sp.). Après les avoir poursuivis toute la nuit dans les arbres grâce aux reflets de leurs yeux, Astre ("de la nuit») les voit disparaître au petit matin dans un "trou dara" (nid d'ara) en haut d'un tronc mort de palmier. Il grimpe au $t$ ronc et penche la tête dans le trou pour apercevoir ses beaux-frères, mais ceux-ci, de l'intérieur du trou d'ara, agrippent sa tête, la tirent, et le décapitent. La tête d'Astre tombe au fond du palmier creux dans le monde souterrain des poissons avec lequel communique le palmier du " $t$ rou dara" et se transforme en racème de parépou (Bactris gasipaes).

"Souffleur de Sarbacane", fils d'Astre, grandit orphelin de père. Adolescent, il apprend que ses oncles maternels, les douroucoulis, ont tué son père et vivent cachés en parasites en haut des quatre poteaux centraux de la maloca en se faisant nourrir par sa mère. Pour venger son père, il décide de les tuer, les fait cuire, les mange et replace leurs crânes en haut des quatre poteaux centraux. Ayant appris son méfait, sa mère se suicide en se faisant passer pour un kinkajou qu'elle enjoint à son fils de tuer. Il la tue et la mange, reprend le nom de son père «Astre » et décide d'errer de par le monde après avoir compris qu'il avait mangé sa mère. 


\section{Seconde partie}

Après de longues aventures, le mythe se poursuit sous l'eau, dans le monde des poissons. Ayant enlevé, amené sur terre et gardé comme épouse une femme poisson (Brycon sp.), le héros part chez son beau-père, Anaconda des Poissons, pour régulariser le rapt. Arrivé dans le territoire de ses alliés, il se fait reconnaître par son beau-père et par ses beaux-frères les poissons comme leur allié "Soleil du Milieu ».

Les poissons jouent au jeu de balle devant leur maloca et invectivent le héros en lui disant "qu'il vient sans genoux "; ils le mettent au défi de venir récupérer " la chair de son père "; le racème de parépou issu de la tête décapitée. Venant rejoindre la partie, Soleil du Milieu envoie la balle de caoutchouc sur le racème de parépou qui se décroche et s'écrase à terre ; les fruits éclatent et libèrent leur noyau. Le héros en récupère un en cachette, l'avale et le rapporte sur terre. D'origine astrale, le noyau brûle l'estomac du héros qui, saisi de violentes diarrhées, le défeque à l'arrière de sa maloca (endroit où le palmier doit aujourd'hui être planté).

Dénoncé auprès d'Anaconda des Poissons par deux tangaras des palmes (Thraupis palmarum) (frères classificatoires de la femme poisson) envoyés de par le monde pour retrouver le palmier volé, le héros perd le premier palmier qu'il avait fait pousser ; son beau-père fait tant pleuvoir que l'eau monte jusqu'à l'arrière de la maloca. Les poissons arrivent avec l'eau, arrachent le palmier, et repartent avec la décrue. Il ne reste que deux petites "sardines" (Aequidens sp.) emprisonnées dans un trou d'eau laissé par le tronc dans le sol. Contre la vie sauve, elles promettent à Soleil du Milieu de faire repousser le palmier à partir d'un morceau de racine qu'elles ont trouvé dans la cavité. Le palmier repousse sous une forme cultivée.

Mais Raie n'en reste pas là et conspire contre celui qu'il considère comme le voleur de sa femme. Aidé par une partie de ses beaux-frères poissons, il demande qu'Anaconda des Poissons lui donne un "parépou de la guerre"; avec les épines du tronc, il confectionne les lances de ses guerriers - aiguillons et dards des poissons. Il fait appeler Soleil du Milieu pour le tuer. En chemin, la femme poisson informe le héros qu'elle était déjà l'épouse de Raie et qu'il lui demandera où se trouve son cœur pour le tuer ; afin d'échapper à la mort il devra montrer sa cheville.

Arrivé chez les poissons, Soleil du Milieu est sommé par Raie de montrer où se trouve son cœur. Il désigne sa cheville, son genou, d'autres articulations et plusieurs parties de son corps où tous les poissons viennent planter, à tour de rôle, leur lance. Laissé pour mort, deux femmes Crevettes décident de lui venir en aide par peur des représailles qu'il menace de mettre à exécution s'il ne revient pas sur terre : assécher l'eau afin de permettre à ses "grands-pères" (= toutes les espèces ichtyophages, des fourmis aux papillons, en passant par les hérons, martins-pêcheurs, etc.) de faire un carnage en dévorant les poissons. Pour fomenter sa vengeance, Soleil du Milieu décide

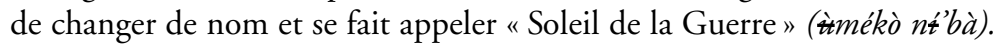

Mis au courant des projets de représailles de Soleil de la Gu er re, certains poissons viennent retirer les lances qu'ils avaient laissées dans le corps dAstre. Soleil crée la loutre qui part à l'orient en dévorant les mains et les pieds des poissons pour ave rtir les "grands-pères" de Soleil de la situation dans laquelle se trouve leur protégé. Ces derniers arrivent en remontant le courant et en dévorant tous les gens d'Anaconda des Poissons qu'ils trouvent sur leur passage. Les survivants ave rtissent les habitants de la maloca d'Anaconda des Poissons et leurs conseillent de fuir. Trop tard, la maloca est déjà entourée par les protecteurs de Soleil de la Gu er re. Le maître de la maloca prie son gendre d'écarter la furie de ses "grands-pères » et il enjoint ses

\section{Dimitri Karadimas}


gens denlever les lances qui restent dans le corps de son gendre; tous s'exécutent sauf Raie.

Les gens de la maloca sont épargnés, mais le héros reste avec la pointe de la lance de Raie fichée dans la cheville. Allongé dans son hamac, il décrit tous les arbres fruitiers de son verger qui, de par son absence, vont se perdre sur terre. Lorsqu'il arrive à évoquer l'umari (Poraqueiba sericea Tul.), la Raie l'arrête et lui demande quel est ce fruit. Le héros promet de le lui faire goûter et de l'inviter à une fête de boisson une fois libéré de son tourment. Raie enlève la pointe de sa lance et Soleil repart sur terre pour préparer la fête. Raie lui demande de lui mettre une provision de masse de fruits à part de celle des autres poissons.

Soleil prépare la fête de boisson à laquelle, avec la crue, les poissons viennent participer. Ces derniers prennent la part qui leur revient et repartent avec l'eau (l'eau boueuse des premières pluies a la même couleur que la boisson préparée avec de l'umari). La part de Raie est laissée en amont par Soleil ; les poissons ont le temps de repartir avant que Soleil n'installe le barrage qui coupe la retraite de Raie. Se sentant pris au piège, Raie remonte la rivière jusqu'à sa source et s'enfouit dans un bourbier pour déguster la masse d'umari préparée par son ennemi.

Soleil cherche Raie mais ne le trouve pas. Il entreprend des travaux de désherbage dans ses jardins et fait brûler des mauvaises herbes qui éclatent sous la chaleur. Raie, pensant que l'été est arrivé (ces plantes éclatent sous l'effet du soleil de l'été), se met à crier victoire de sa cachette supposant qu'il ne lui reste plus longtemps à attendre avant les pluies. Soleil le repère, fait éclater d'autres herbes, et aperçoit les yeux de Raie qui dépassent du bourbier. Soleil le rejoint et lui demande où se trouve son cœur. Celuici lui indique que, n'étant pas poltron, il n'a pas peur de mourir : «il est là bien au centre". Soleil pique Raie avec sa lance pour qu'il se relève; une fois debout, il lui plante sa lance dans le cœur. Il le transporte jusqu'au foyer et le brûle.

De ses cendres naissent plusieurs plantes (l'ortie contre les douleurs articulaires, une liane à venin, et «l'herbe de la raie " Pothomorphe peltata appliquée sur les genoux des enfants qui tardent à marcher, ou qui sont "faibles des genoux »).

\section{Une interprétation astronomique}

Le résumé que nous venons de faire de la geste de "Souffleur de Sarbacane", nécessite plusieurs commentaires. La lecture du mythe peut se faire selon des approches diverses; sociale, rituelle, temporelle voire psychologique. Nous ne prendrons en compte dans notre analyse que son aspect temporel; le découpage de l'année associé aux crues et décrues qu'il énonce, ainsi que les saisons et les périodes de fructification de certains arbres.

La pre m i è re partie du mythe traite de l'origine du parépou, qui est la tête transformée dAstre, alors que la seconde partie renvoie à la récupération d'une partie de cette tête paternelle perdue, pour en faire le premier palmier terrestre. Nous reviendrons plus longuement sur l'association entre cervelle et parépou, mais nous pouvons dès à présent noter que l'association entre tête et racème n'est pas un fait isolé dans la région et se retrouve également, selon Reichel-Ddmatoff (1997: 237, n. 14), chez les Desana (groupe de langue Tukano oriental) ${ }^{4}$ :

4. Chez les Desana, le mythe de l'origine du parépou est, à quelques variantes près, identique à celui des Miraña. Nous re n voyons le lecteur à $O$ mito de Gäipayã e a origem da pupunha dans le recueil ... / ... 
"Comparaciones similares se hacen con frutos de la palma pupunha o pejibaye, a cuyo respecto algunos informantes señalaron que aquellos representan un ventriculo kaë [de la cervelle], en tanto que un racimo de frutas forma un solo cerebro.»

Pour les Miraña, c'est la même association entre racème de parépou et cerveau qui est utilisée dans le mythe (cf. Karadimas 1997). Ainsi, toujours dans le mythe, l'association entre parépou et cervelle pose celle-ci comme origine de celui-là.

Le fils d'Astre reprend l'identité paternelle en même temps qu'il accomplit la vengeance du meurtre de son père.

Les Miraña désignent la lune et le soleil par le même terme (nílbà : «astre») et les distinguent par un qualificatif qui se réfere à la valeur nocturne ou diurne de l'astre. Nous avons donc affaire, dans le mythe, à une succession entre deux personnages portant le même nom mais qui ne représentent pas le même astre.

J'ai montré ailleurs (Karadimas 1999) que la première partie du mythe renvoyait à la disparition occidentale de l'astre lunaire précédé par la constellation d'Orion. Les quatre douroucoulis (Aotus sp.) - ou «singes nocturnes»-, évoquent les quatre étoiles du trapèze d'Orion qui disparaissent derrière un affleurement rocheux à l'horizon occidental (l'escarpement de Araracuara), alors que le père du héros est l'astre lunaire. Cette première partie du mythe rend compte d'un phénomène astronomique lié à la constellation d'Orion pendant lequel elle est poursuivie par la lune durant une moitié de l'année. Par ailleurs, Orion possède la particularité d'encadrer l'équateur céleste qui passe en son centre : il s'agit donc d'une constellation qui est associée aux points cardinaux Est et Ouest. Ce phénomène qui relie la lune et Orion se déroule à partir du solstice de décembre jusqu’à quelques jours avant le solstice de juin. La constellation disparaît du ciel nocturne avant l'arrivée du soleil ; elle correspond donc, durant cette période, à l'astre nocturne. Cette première phase de la constellation débute et se termine avec les solstices, mais elle culmine autour de l'équinoxe de mars, moment de l'année où la lune (en pleine lune) suit un trajet d'est en ouest qui se superpose à l'équateur céleste. L'astre nocturne est dans l'alignement qui passe au centre de la constellation d'Orion et termine sa course au même point d'équinoxe à l'horizon occidental (équinoxe lunaire). Le mythe rend compte de ces observations astronomiques en présentant la décapitation d'Astre (nocturne) par les quatre singes cachés dans un "trou d'ara" (Araracuara), en haut d'un palmier creux (le canal formé par le Caquetá dans cet affleurement rocheux). La tête tombe dans le monde souterrain des poissons et devient un racème de parépou.

Nous re p roduisons ici trois motifs de poterie issus de pièces archéologiques du Nariño (Période Capuli, Andes colombiennes) (cf. Fig. 1 et 2). Nous avons placés artificiellement deux de ces motifs en superposition au centre de celui de singes placés tête-bêche et encadrés par quatre représentations d'étoiles

de mythes que les Desana ont eux-mêmes publiés sous le titre de Antes o mundo não existia (Pãrōkumu \& Kêhíri 1995 : 169-177). 


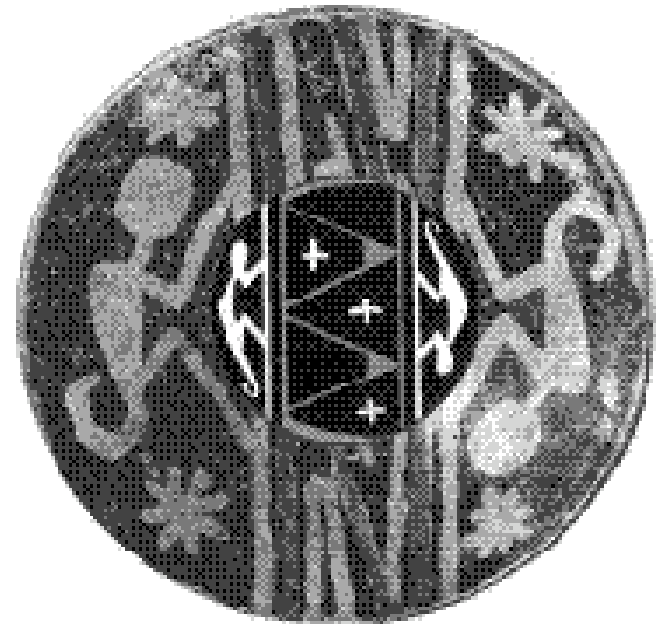

Fig. I

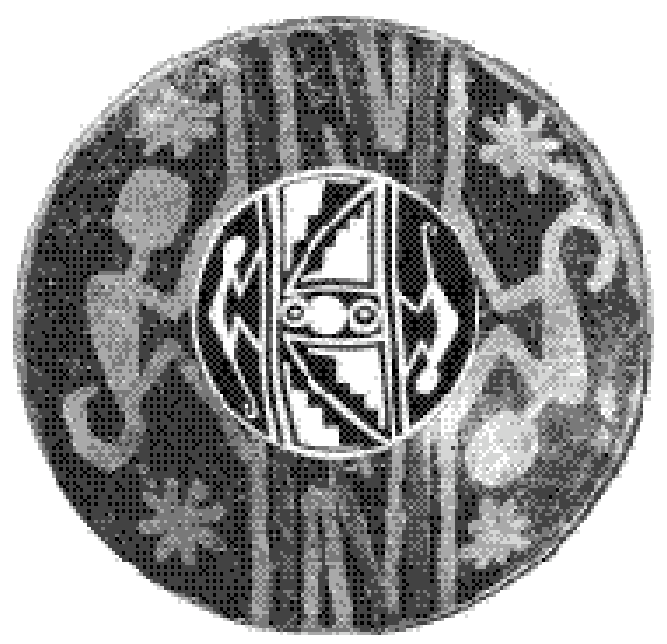

Fig. 2

(cf. Karadimas 1999). La même poterie est utilisée pour les deux compositions; seul le motif central change alors que la structure générale reste identique sur les trois poteries. Sur la premièrecombinaison, la constellation d' Orion apparaît dans son ensemble, les étoiles qui forment le trapèze et le Baudrier suivent assez fidèlement la disposition des étoiles de cette constellation comme elles se présentent dans le ciel (cf. infra pour une illustration de la constellation). La seconde combinaison montre que les trois étoiles disposées suivant une certaine inclinaison dans le premier cas peuvent également être figurées alignée horizontalement: il n’y a que l'étoile centrale du Baudrier qui est représentée différemment aux deux autres étoiles du Baudrier (epsilon d' Orion, possède un magnitude supérieure aux deux autres étoiles du Baudrier : nous reviendrons sur ces détails astronomiques qui s'expriment sur des pièces archéologiques et dans l'iconographie des masques contemporains). Il faut retenir de ces motifs de poterie que le trapèze d'Orion est traité dans l'iconographie à la périphérie de la bande centrale, alors que le Baudrier a été placé à l'intérieur de cette bande. Cette opposition de traitement entre centre et périphérie implique que le Baudrier et le trapèze d'Orion peuvent être appréhendés et rendus séparément par les pensées indigènes (nous re $t$ ro $u$ ve rons ce traitement séparé sur l'iconographie des masques contemporains).

Les motifs des singes sont absents dans le rituel de la bière de parépou, et semblent avoir laissé la place à celui de la raie. C'est l'étude des phénomènes saisonniers présents dans le mythe qui va nous permettre de poursuivre l'analyse.

L'équinoxe de mars est également la période de l'année pendant laquelle a lieu la maturation la plus abondante des fruits du parépou qui permet l'organisation $\mathrm{du}$ rituel; raisons pour lesquelles ces constatations saisonnières sont présentées dans le mythe auquel les Miraña font référence pour illustrer et expliquer le rituel. Cette fête est donc associée en tout premier lieu à l'équinoxe de mars ainsi qu'à la constellation d'Orion et à la lune.

Durant la seconde partie de l'année (du solstice de juin à celui de décembre), Orion ne précède pas la lune à l'ouest, mais il est lié à l'est où il précède le lever du 
soleil. En un sens, les deux phases sont en opposition complémentaire et partagent l'année en deux parties. Première phase: Orion précède le coucher de lune à l'horizon occidental; seconde phase : Orion précède le lever du soleil à l'horizon oriental.

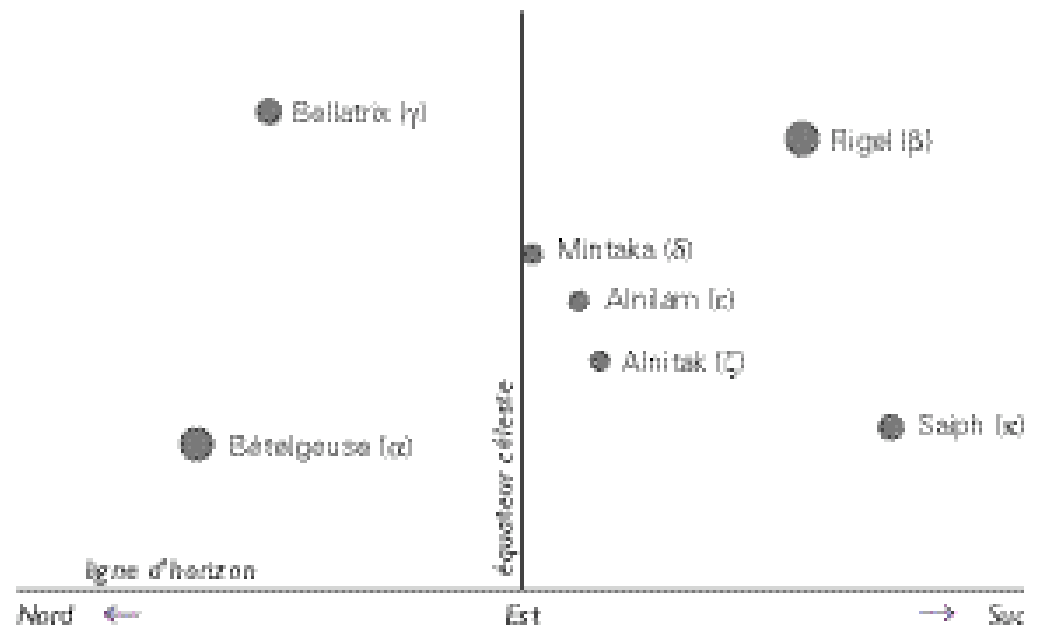

Fig. 3. Lever héliac d'Orion

La première partie du mythe décrit donc un trajet dans le ciel d'est en ouest vers l'inframonde (la tête décapitée de lune qui part dans le monde des poissons), alors que la seconde décrit un trajet de l'inframonde vers le monde du dessus (trajet souterrain, ou aquatique d'ouest en est).

Les deux personnages principaux de cette seconde partie du mythe sont Soleil du Milieu et Raie. Si la raie est utilisée dans ce mythe pour marquer l'antagonisme avec Soleil du Milieu, elle occupe - dans son rapport avec Astre - la même place que les quatre singes dans la première partie du mythe, si ce n'est qu'ils ne sont pas alliés. En d'autres termes, Raie occupe vis-à-vis de Soleil du Milieu la même place que les quatre singes vis-à-vis de son père; il devrait donc s'agir d'une évocation de la constellation d'Orion dans sa seconde phase, lorsqu'elle est associée au lever du soleil.

La place relative des deux protagonistes de la seconde partie du mythe recouvre en fait une association plus fondamentale qui, dans l'épopée, prend son origine avant l'engendrement de Soleil du Milieu par Lune. L'épisode mythique qui précède la décapitation de Lune par ses beaux-frères, décrit comment la femme kinkajou, lors de ses premières règles, sauve Lune de la furie de ses consanguins en le recueillant clandestinement dans sa chambre de réclusion. Dans un précédant travail (Karadimas 1997), j’avais évoqué la possibilité que la raison de cette trahison de la femme kinkajou envers ses consanguins soit due au fait qu'elle ait été victime d'un abus sexuel de la part de ses frères ${ }^{5}$. L'origine des règles serait le résultat de cette relation incestueuse, à l'occasion de laquelle, de surc roît, aurait été placé dans son

5. Les Miraña attribuent un comportement incestueux à plusieurs animaux, et plus particulièrement aux singes ; catégorie dans laquelle les Miraña classent le kinkajou. 
ventre un être qui en portera les stigmates, puisqu'il est issu en partie du sang menstruel et de la semence de ses quatre géniteurs. La place que prennent ensuite les quatre singes dans la maloca en se faisant nourrir par leur sœur est significatif de ce comportement incestueux ; à cela vient s'ajouter le fait que la mère se fait volontairement tuer lorsqu'elle apprend le meurt re de ses frères par son propre fils. Les quatre singes occupent donc auprès de leur sœur kinkajou la place de leur beaufrère Lune aussi bien du point de vue sociologique que spatial.

Pour les Desana, à l'ethnographie desquels nous avons déjà eu recours, la première raie est issue d'un placenta (Reichel-Ddmatoff 1973: 55). Ce rapprochement semble être fondé sur une analogie formelle entre ce poisson et le placenta qui, une fois déplié après sa chute, prend une forme de disque ou de galette. Associé au cordon ombilical, il n'est pas sans rappeler une raie et sa queue, d'autant plus qu'il est pourvu d'aspérités rugueuses comme l'est la face dorsale de la raie.

Pour les Miraña, le placenta est considéré comme le "masque", le "visage féroce " (gwás̆à timì) du foetus, ou encore "l'ombre de l'enfant " (= du foetus) (tsíménè nà:ßénè). L'inverse est également vrai et les masques - voire même le visage pour la tête lorsqu'elle devient trophée - sont également perçus comme des placentas. La "face " ou le "visage féroce " est aussi une formulation utilisée pour qualifier les masques du rituel du parépou ${ }^{6}$. Pour les Miraña toujours, le placenta et le cordon ombilical proviennent d'un élément autre (animal ou humain) dont le père s'est nourri et qui, une fois dans le ventre maternel, tente de reprendre vie en participant à la création du fotus avec l'espoir d'en prendre possession lors de sa naissance (cf. Karadimas 1997). Il s'agit d'un double, sauvage, animal et féroce de la personne, mais dénaturé car avorton et qui, pour cette raison, est enterré après l'accouchement et auquel est attribuée une paternité autre que celle de l'enfant. Dans les mythes miraña, le placenta d'une entité se transforme toujours en un autre être, végétal ou animal (la poule est ainsi le placenta du chien, le tinamou celui du jaguar, etc.).

Les Andoque partagent, dans des traits principaux, le même corpus mythologique que les Miraña - situés près de Araracuara, ils sont leurs voisins immédiats. La version andoque de l'origine du parépou est identique à celle des Miraña si ce n'est que Soleil prend la figure d'un cabiai (Hydrochaeris hydrochaeris) pour évoquer le séjour subaquatique del' a st re diurne. Soleil a les mêmes déconvenues ave c une raie qui le pique au genou, l'invite à une fête de boisson une fois rétabli, etc. (Landaburu \& Pineda 1984 : chap. 10). Les Andoque attribuent l'origine de la raie à la tête d'un anaconda immature qui s'éve rtue à tuer un tapir. Ac c roché par une extrémité de son corps au fond de l'eau, l'anaconda tente vainement de retenir le tapir dans ce milieu aquatique. Ce dernier, après plusieurs va-et-vient vers la s u rface, parvient à en faire sortir sa tête qu'il maintient hors de l'eau, puis son corps, étirant à chaque fois un peu plus le corps de l'anaconda. Sectionné par une racine coupante contre laquelle le tapir passe à plusieurs reprise alors qu'il s'extrait

6. Pour une compréhension plus large des rapports entre le masque et le placenta chez les Miraña, on se reportera aux pages 259, 340 et 716 passim de ma thèse (Karadimas 1997). Il semble que Jean-Pierre Chaumeil, dans son article (2000-2001), soit parvenu à des conclusions analogues de façon indépendante. 
du milieu aquatique, l'anaconda meurt et se divise en deux parties : après putréfaction, sa tête devient la grande raie, et les liquides produits forment un bourbier (le mythe ne dit pas ce qu'il advient de l'autrepartie du corps sectionné). La raie se fait porter, comme un nouveau-né, dans une bande de portage par un personnage qui, comme dans le mythe miraña, travaille dans son jardin et la repère par les cris qu'elle pousse pensant que l'été est arrivé (ibid: 182 sq.).

La forme même de ce mythe laisse penser à un accouchement au cours duquel la triade fotus/cordon ombilical/placenta est occupée par le tapir, l'anaconda (qui, comme le cordon ombilical, finit sectionné) et la raie, émanation de la tête de l'anaconda (inversant toutefois avec le nouveau-né sa place dans la bande de portage où elle le remplace). Il est d'ailleurs fort possible que cette partie de la mythologie andoque soit d'origine miraña puisque, d'après les Andoque, " $L a$ raya habla en miraña" (ibid.: 189, n. 29) 7 .

Contrairement aux Desana et aux Andoque, les informateurs miraña avec lesquels j'ai travaillé ignorent l'origine de la première raie: simplement ils estiment qu'elle ne doit pas être consommée car elle rend belliqueux et paresseux, mais surtout, sa consommation induit des comportements incestueux. Ils pensent en effet que sa forme, proprement monstrueuse, doit être le résultat d'une telle union, et ils ne la classent d'ailleurs pas parmi les poissons. Avec d'autres animaux à robe tachetée, la raie, chez les Desana, appartient également à la catégorie des animaux incestueux ${ }^{8}$.

Il est ainsi fort probable que, dans le mythe miraña, le personnage Raie soit issu de l'union incestueuse de la femme kinkajou et de ses frères. À la suite de cette première union, Lune la prend comme épouse et engendre Soleil du Milieu. Dans la matrice maternelle se trouveraient donc un foetus et son placenta-cordon ombilical, respectivement Soleil du Milieu et Raie. Ainsi, un mythe tupi de l'origine de Jurupari présente la même structure de base que le mythe miraña, si ce n'est qu'il remplace la femme kinkajou par une mère sarigue, dotée d'une matrice double, dans laquelle deux pères vont, chacun à leur tour, engendrer une progéniture dont l'une des deux deviendra Soleil (voir, à ce sujet, Lévi-Strauss 1971). Mammiferes marsupiaux (donc aplacentaires), les sarigues ne produisent pas de placenta et, dans ce cas, le mythe leur fait engendrer des jumeaux (la paire Soleil / Lune). En revanche, dans le cas où il faut considérer le rapport entre Lune et Soleil comme transgénérationnel (père / fils), le « double » de Soleil n’est plus son jumeau Lune, mais une entité placentaire. Or les Baniwa (de langue arawak et

7. Jon Landaburu, ethnolinguiste des Andoque à qui l'on doit, avec Roberto Pineda, ce recueil de mythes, pense également que cet épisode mythique pourrait être d'origine miraña (communication personnelle).

8. Reichel-Dolmatoff 1997: 165. Le fait de classer comme incestueuses les espèces tachetées semblerait p rovenir d'une extrapolation d'un mythe très répandu qui relate comment la sœur de Lune, pour reconnaîtrel'amant anonyme qui la visite chaque nuit, lui couvre une partie du visage de genipa (teinture corporelle temporairement indélébile qui ox yde la peau). Reconnu le lendemain matin par les tâches qu'il porte sur le visage, Lune s'enfuit de honte dans le ciel. Ces tâches sont encore visibles sur le "visage " de la lune et permettraient donc de reconnaîtrele caractère incestueux d'une espèce. Chez les Andoque, c'est la belle-sœur de Lune qui, sur les recommandations de son mari Soleil - frère jumeau aîné de Lune - agit de la sorte pour reconnaîtreson amant. 
voisins septentrionaux des Tukano du Vaupès) voient justement dans le placenta de Jurupari l'origine de la raie (Lévi-Strauss 1966 : 264, n. 1). Dans le cas où une des valeurs accordées à Jurupari est Soleil, la raie doit donc être considérée comme le placenta du soleil, elle représente son double et, sur le plan astronomique, il devrait s'agir d'Orion?.

Nous pouvons donc supposer que la relation qui lie Soleil du Milieu à Raie est de l'ordre de l'aérien et du chtonien (le placenta étant enterré ou rendu à la rivière, Raie peut être considéré comme un personnage de l'inframonde); avec cette particularité qu'il s'agit là d'une relation antagoniste. Raie représente donc le double chthonien de Soleil du Milieu et, s'il s'agit de la constellation d'Orion, cette dernière est pensée comme un "double chtonien » du soleil, ce qui, quant au mode astronomique, n'est pas incongru (Orion occupe, dans le ciel nocturne, la place du soleil dans le ciel diurne). Cette relation est exprimée par un code sociologique dans le mythe : vis-à-vis de Soleil du Milieu, Raie occupe la place de co-époux auprès de la Femme poisson. Cette place en fait un sorte d'équivalent, de double, un alter ego, demi-frère par la mère de Soleil du Milieu ${ }^{10}$.

Nous pouvons donc supposer que la dispute entre les quatre singes et Lune dans le premier épisode se poursuit dans le second par un antagonisme entre leur descendance respective - Raie et Soleil du Milieu. Les indications temporelles présentes dans le mythe permettent de confirmer cette hypothèse, tout comme il est possible de montrer que l'utilisation par la pensée mythique de la figure de la raie renvoie à certaines particularités de son anatomie.

En effet, le nom complet de la raie est « raie-jaguar » qui est le nom miraña de la plus grande des espèces de raie rencontrées dans le Caquetá (sous famille des Potamotrygon). Lassociation avec le félidé proviendrait du fait que sa robe est tachetée comme celle du jaguar et que sa piqûre est la plus douloureuse de toutes. Ces " tâches" sont en fait des ocelles que la raie possède sur sa face dorsale, constitués chacun par de petits cercles concentriques, et qui lui servent de camouflage lorsqu'elle s'enterre dans les fonds sablonneux. Ces ocelles sont répartis plus ou moins symétriquement sur chacune des « ailes» de la raie. En prenant en compte les deux plus grands de ces "faux yeux ", un par aile, ils occupent les sommets d'un trapèze dont les véritables yeux formeraient la base la plus courte. En ce sens, les ocelles et les yeux de la raie forment un trapèze qui, s'il est associé au soleil, évoquerait la seconde phase de la constellation d'Orion, c'est-à-dire lorsqu'elle est ascendante, la base la plus courte du trapèze dirigée vers le haut. Mon hypothèse est donc que faux et vrais yeux forment sur Raie les stigmates incestueux laissés par ses géniteurs les quatre singes.

9. Pour les Tikuna, voisins méridionaux éloignés des Miraña, qui possèdent également une fête avec plusieurs masques d'animaux, le personnage de la raie joue un rôle similaire : elle est le placenta d'un foetus qui a été retiré immature de la matrice maternelle. Cette raie-placenta tikuna joue alors un rôle de mère vis-à-vis du foetus; elle le nourrit et le couve jusqu’à sa maturité (Nimuendajú 1952 : 120).

10. En tant qu'allié d'alliés, Raie possède un caractère consanguin vis-à-vis de Soleil du milieu. Dans le mythe, cette caractéristique est marquée par l'utilisation d'un type sociologique prop re: «l'ami » (gókoò) utilisé par ceux qui partagent un ensemble de biens. Je remercie France-Marie Casevitz pour a voir attiré mon attention sur cet aspect du mythe. 


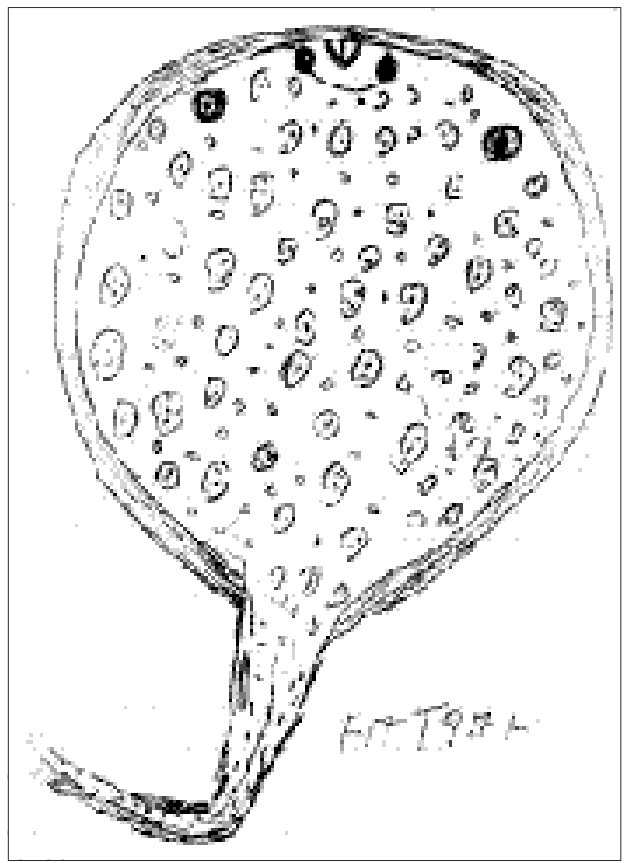

Fig. 4 Raie dessinée par un Trumai (Haut Xingu) (Monod-Becquelin 1993)

Nous reproduisons ici à titre dillustration le dessin d'une raie fait par un Trumai du Haut Xingu sur lequel semblent apparaître les yeux et les ocelles formant trapèze (in MonodBecquelin 1993: fig. 103). Il s'agit juste de montrer par ce dessin que cette particularité dans la robe de la raie n'échappe pas à l'observateur indigène. Il existe toutefois une autre possibilité pour rend re compte du fait que la raie est perçue comme une espèce à "quatre yeux". En prenant alternativement chacune des faces - ventrale et dorsale - du sélacien, elles forment chacune pour elle-même un visage. La face dorsale est exempte de bouche, laquelle se trouve reléguée sur la face ventrale. La bouche de la raie forme une ligne courbe dirigée vers le haut qui fait penser à une bouche "souriante ». Placés légèrement au-dessus de l'ouverture buccale, les deux narines de l'animal constituent deux trous nettement distincts. Lorsque cette face ventrale est anthropomorphisée, c'est-à-dire que l'on tente de la rapprocher formellement d'un visage humain - processus qui est communément réalisé puisqu'il est une opération cognitive majeure de connaissance (cf. Karadimas 1997) -, ces narines occupent la place que prendraient des yeux sur un visage. Chacune des faces posséderaient ainsi ses prop res " yeux ». Sur le dessin trumai reproduit ci-dessus, l'artiste a tracé entre les deux yeux du haut une ligne courbe qui les relie. Or cette particularité est absente de toutes les espèces de raies d'eau douce du bassin amazonien. Il ne nous est pas possible d'affirmer avec certitude que l'a riste a voulureprésenter l'ouverture buccale de la raie en la faisant apparaître alors qu'elle est normalement cachée lorsque la seule face dorsale est prise en compte. Il est fort probable que les deux possibilités - celle d'associer les deux faces ou les yeux et les ocelles - soient complémentaires pour exprimer le fait que la raie possède "quatre yeux ".

Revenons maintenant à l'analyse du déroulement du mythe. Nous n'allons pas parcourir l'ensemble des données saisonnières décrites par le mythe, mais nous contenter de sa dernière partie lorsque Soleil tue Raie.

Lorsque les poissons sont invités à boire du jus d'umari, l'épisode se situe pendant sa période de fructification qui s'étend sur plusieurs mois, de décembre à avril (Guyot 1975 : fig. 6). Les premières fortes pluies de mars entraînent des crues qui se prolongent jusqu'à la fin du mois d'avril, temps qui correspond également à la fin de la période de l'umari. Soleil du Milieu voit Raie remonter le courant de la rivière. Suivant un code astronomique, il pourrait s'agir d'Orion 
qui passe sous la terre d'ouest en est, une évocation d'Orion vespéral qui a déjà atteint l'horizon occidental au début de la nuit. De mai jusqu'à la première semaine de juin, Orion n'est pratiquement plus visible à l'horizon occidental car il est à la fois trop rapproché du coucher du soleil et partiellement caché par l'horizon surélevé des contreforts rocheux de Araracuara.

Dans le mythe, Soleil du Milieu passe ensuite plusieurs jours à chercher son ennemi Raie sans arriver à l'apercevoir : il est fort probable que nous soyons en présence d'une évocation de la disparition momentanée d' Orion du ciel nocturne jusqu'au solstice de juin. Ainsi, en poursuivant les indices spatio-temporels donnés dans le mythe, lorsque Raie remonte la rivière jusqu'à sa source, c'est sans doute au cours d'eau souterrain qui va d'est en ouest qu'il est fait allusion (chemin parcouru à contre-courant dans le dessous du monde par les corps célestes). Raie poursuivi par Soleil du Milieu semble donc se diriger vers l'est. De plus, le héros entreprend des travaux dentretien de ses jardins et fait croire involontairement à Raie que l'été est déjà de retour. Le héros aperçoit enfin Raie grâce à ses yeux qui trahissent sa présence en dépassant du bourbier dans lequel il s'est enfoncé.

Suivant la compréhension astronomique du mythe, il s'agirait, après le solstice de juin, d'Orion qui réapparaît partiellement à l'est par les deux étoiles de la base la plus courte du trapèze (Rigel et Bellatrix). Ces deux étoiles formeraient, suivant notre hypothèse, les yeux de la raie. Sur le plan astronomique, le soleil est alors latéralement au plus loin sur la ligne d'horizon par rapport à son lever équinoxial: au moment où il fait "disparaître" Orion avec les autres étoiles. Le mythe transcrit l'événement par le fait qu'il pique Raie et le force à se lever (dans les premiers jours de juillet la constellation est de nouveau « debout », c'est-à-dire entièrement visible). Nous allons voir pourquoi Soleil du Milieu doit attendre l'équinoxe de septembre pour tuer son ennemi. Le héros décide de tuer Raie en lui plantant sa lance en plein cœur, c'est-à-dire, pour les Miraña, au centre de celle-ci (ce qui correspondrait à l'équinoxe de septembre).

L'ensemble de la description couvrirait ainsi un phénomène qui a lieu entre les deux périodes solsticiales, dont le point culminant est le moment de la seconde fructification du parépou à l'équinoxe de septembre.

Il nous faut toutefois revenir sur l'épisode du mythe dans lequel Raie dévoile l'endroit où se trouve son cœur. En effet, celui-ci semble au premier abord quelque peu énigmatique, puisque Raie se condamne en agissant de la sorte. Cet apparent abandon dans la lutte qui l'oppose à Soleil du Milieu doit être quelque peu nuancé par le fait que la raie possède ailleurs dans les mythologies amérindiennes des dons d'esquive dont la dote son épaisseur minime lorsqu'elle se présente de profil. Ainsi, suivant un mythe des Tillamoock d'Amérique du Nord analysé par Claude Lévi-Strauss (1971: 487) : «Les Tillamoock évoquent un combat singulier entre la Raie et le Cervidé $\left(\mathrm{M}_{779}\right.$; E. D. Jacobs, p.11). Ce dernier se croyait sûr de vaincre, car son adversaire était si large que toute flèche ne pouvait manquer de l'atteindre en plein corps. Mais la Raie sut chaque fois se mettre de profil au bon moment, et, quand vint son tour de tirer, ce fut le Cervidé qui mourut. » Nous aurions dans le mythe Miraña, avec ce qu'il faut 
bien considérer comme un défi de Raie envers son ennemi, l'équivalent inverse de cette certitude affichée par le cervidé dans le mythe nord-américain. Raie dans le mythe miraña semble sûr de ne pas être touché parce que Soleil le voit de profil (position du soleil au solstice de juin par rapport à Orion). Soleil est donc obligé de s'approcher pour le piquer afin qu'il se relève ; c'est-à-dire qu'il se présente de face. Le seul moment où cette conjonction a lieu est lorsque le soleil parvient à l'équinoxe et la constellation d'Orion au firmament; à ce moment, le soleil est en face de la constellation d'Orion et, dans le langage mythologique, Raie ne peut plus se mettre de profil pour échapper à son agresseur. Or la variante entre les deux types de mythes relève également d'une opposition liée au déplacement du tireur : dans le cas nord-américain, c'est le cervidé qui reste immobile alors que c'est la raie qui pivote. Dans le mythe miraña, Raie reste immobile et Soleil s'approche de sa cible pour l'obliger à pivoter et se présenter de face (chemin du soleil en solstice vers le soleil à l'équinoxe).

Les mythologies nord-américaines sont aussi celles où l'on retrouve le plus souvent la figure de la raie comme personnage céleste et "plusieurs [mythes] précisent que la Raie demeura au ciel comme un des animaux retardataires qui périrent et furent changés en constellation. Les Quinault (de la côte Nord-Ouest) appelaient une constellation /djagage'h/ "la Raie", qui était peut-être Orion (Olson 2, p. 178)» (ibid. : 487). Bien que l'auteur revienne un peu plus loin pour conclure que la localisation de la constellation de la Raie serait « au voisinage de la Grande Ourse [ce qui] paraîtrait plus vraisemblable que près d'Orion " (ibid. : 492), il nous semble au contraire que, dans le mythe miraña, la Raie occupe la place d'Orion ${ }^{11}$. Enfin, et en Amérique du Sud cette fois, "Les Tumupasa [Ese'eha: Takanan de Bolivie] croient que les raies sont les couvre-chefs des esprits de l'eau, et qu'elles symbolisent la lune " (ibid. : 493), ce qui nous ramène sur le terrain des masques des esprits dans le rituel du parépou, et de leur compréhension astronomique.

Fort de ces conclusions de mi-parcours, il devrait maintenant être possible de rendre aux masques du rituel leurs rôles respectifs et de retrouver ces déductions sur leurs motifs, pour nous permettre de conclure positivement quant à la place prépondérante tenue par la constellation d'Orion dans le rituel lors de sa seconde phase liée à son lever oriental et au soleil.

\section{Les motifs du masque de la Raie}

Les masques miraña présentés lors du rituel relèvent du même type que ceux des Yukuna et des Makuna (Van der Hammen 1992, pour les Yukuna ; Århem 1998, pour les masques makuna). Nous reproduisons ici trois masques recueillis chez les Miraña du Caquetá en 1992.

11. Il s'agirait de démontrer comment l'argument développé par Claude Lévi-Strauss (ibid: 492) concernant la corrélation entre les constellations non nommées et toujours présentes dans le ciel nocturne, qui lui font choisir la Grande Ourse plutôt qu'Orion en Amérique du Nord pour la constellation de la Raie, n'est pas applicable en Amérique du Sud à hauteur de l'équateur où l'accent est justement mit sur l'absence d'Orion, même pour un laps de temps relativement court, du ciel étoilé. 


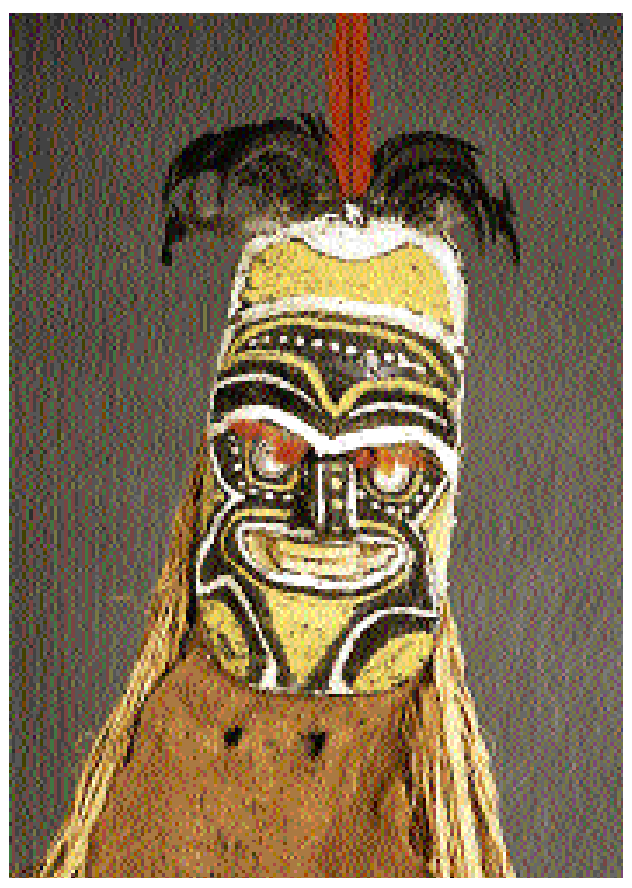

Fig. 5 Masque miraña

du "Maître des Animaux" ou "Soleil du Milieu"

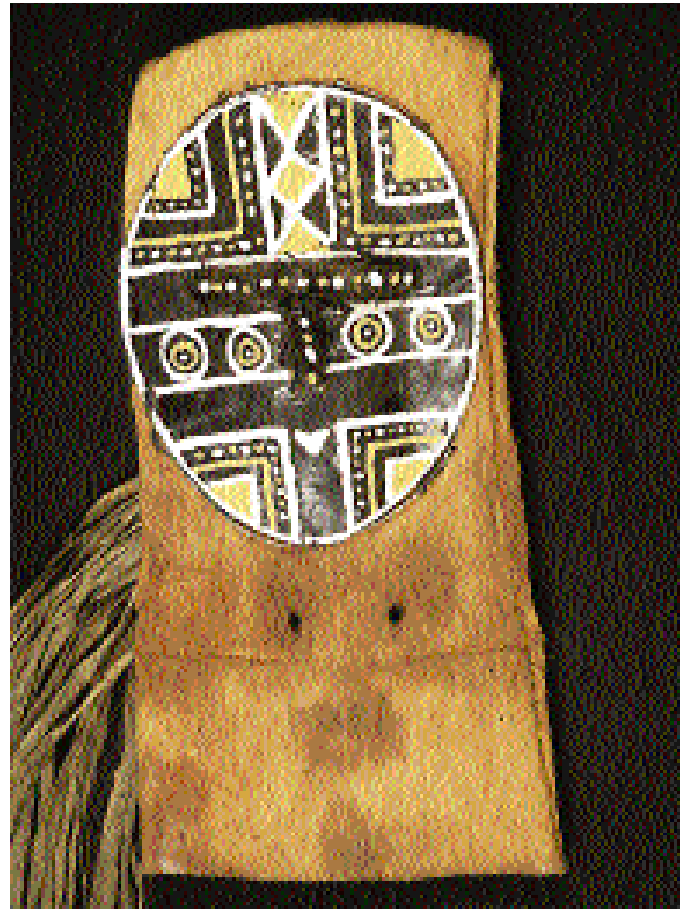

Fig. 6 Masque miraña du "Maître des esprits de l'eau" ou "Raie" 
Le premier (Fig. 5) représente le maître des animaux (mé: $̂$ ) qui est également Soleil du Milieu. Ce type de masque possède une visière ou un diadème de plume en haut du visage qui indique son identité solaire. Ici, la plume caudale d'ara serait une indication d'un rayon solaire tout comme il est l'indice du premier nom du héros "Soleil du Milieu » évocateur du soleil à l'équinoxe et le rictus laissant apparaître la dentition, une référence à l'autre nom du héros "Soleil de la Guerre" (nous ne reviendrons plus sur ce premier type de masque).

Les deux autres (Fig. 6 et 7) sont désignés par les Miraña comme des représentations du maitre des esprits de l'eau et seraient, suivant notre analyse et selon d'autres informateurs miraña, des représentations du masque de la Raie. Il nous faudrait cependant pouvoir étudier ces motifs sur plusieurs masques pour en relever les éléments constants. Nous utiliserons ceux présentés dans l'ouvrage de M. C. van der Hammen (Fig. 8 à 17), que nous supposons être Yukuna puisque l'auteur n'en propose pas d'analyse ni de commentaires, et dont il se sert, dans son ouvrage, comme illustration de début de chapitre.

La première caractéristique d'une partie de ces masques est de posséder quatre "yeux ", alignés, et constitués pour la plupart par des cercles concentriques. Les exceptions sont toutefois importantes et nous permettent de reconnaitre l'évocation du personnage Raie qui, loin de posséder quatre yeux, n'en est pas moins doté, comme nous l'avons vu, de deux grands ocelles sur sa face dorsale. Lorsque nous avons évoqués la possibilité (cf. supra) que la face ventrale de la raie soit anthropomorphisée, c'est-à-dire que la bouche et les narines forment un «visage souriant ", cette éventualité pourrait être évoquée par les masques des figures 11, 15 et 17, où la bouche recourbée vers le haut est placée sous les " yeux » de la face ventrale du sélacien (ses narines).

Si nous prenons en considération ce motif des quatre "yeux" sur le masque de la figure 8, il apparait clairement que les deux yeux les plus rapprochés ne sont pas identiques aux deux autres. Le masque de la figure 8 est la reproduction du motif d'un masque d'un rituel qui a eu lieu en 1946, et qui est illustré sur une photo dans l'ouvrage (Van der Hammen 1992). La première paire représenterait ainsi les yeux de la Raie (du même type que ceux des caïmans ou de certains reptiles et félidés, avec un iris vertical en forme d'amande dans un œil circulaire), alors que les deux autres motifs renverraient aux ocelles qui sont, sur la raie, des cercles concentriques. Les autres masques, contemporains, ne font plus cette distinction - à l'exception de la figure 17 -, et réduisent l'ensemble à des cercles concentriques (la figure 14 réintroduirait partiellement ce genre de distinction).

Il existe pourtant un problème lié à ce type de représentation: les quatre "yeux» sont tous alignés sauf sur le masque de la figure 12, où ils sont disjoints et répartis en carré (comme le sont les quatre poteaux centraux de la maloca où les quatre douroucoulis trouvent refuge dans le premier épisode mythique). Sur la raie, les yeux et les ocelles forment un trapèze, comme le font d'ailleurs les étoiles de la constellation d'Orion. Il faut donc en conclure que ces quatre " yeux " peuvent être représentés sous une forme ou sous une autre, soit en trapèze (ils s'ac- 


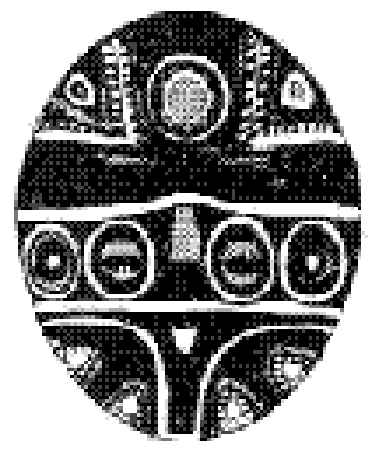

Fig. 8

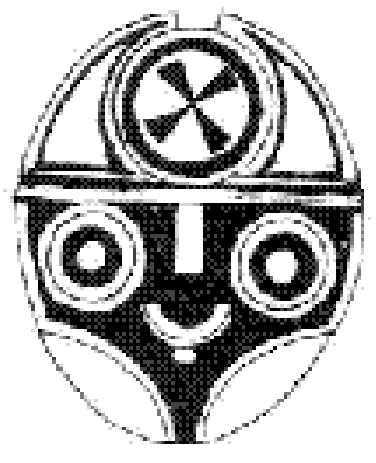

Fig. II

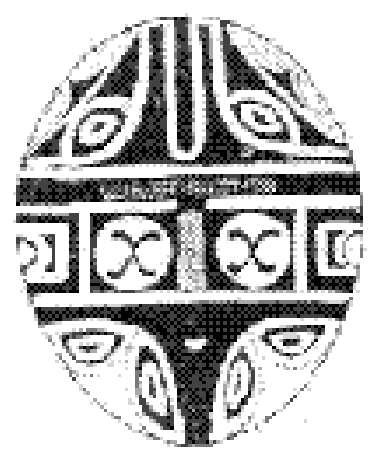

Fig. 14

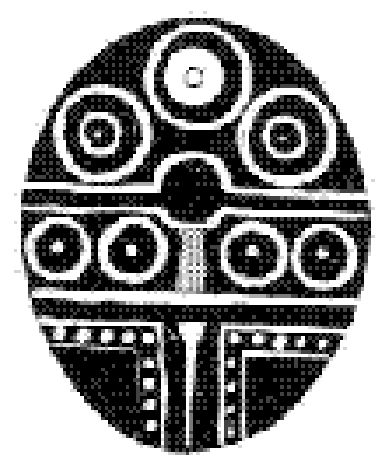

Fig. 9

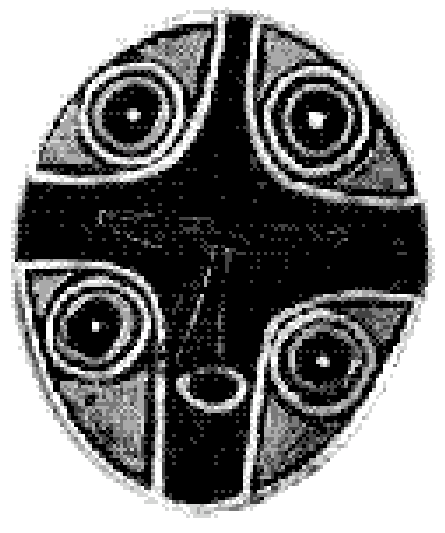

Fig. 12

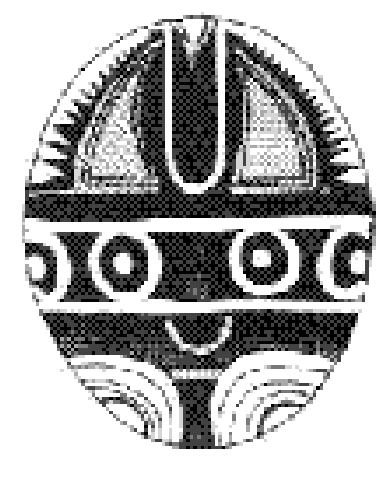

Fig. 15

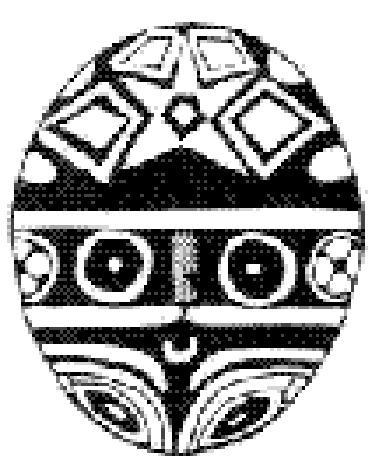

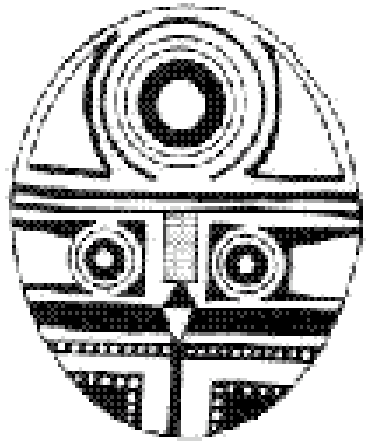

Fig. 10

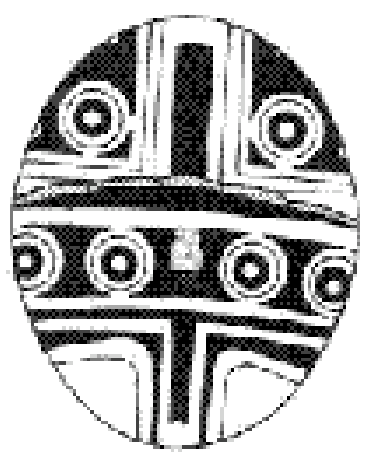

Fig. 13

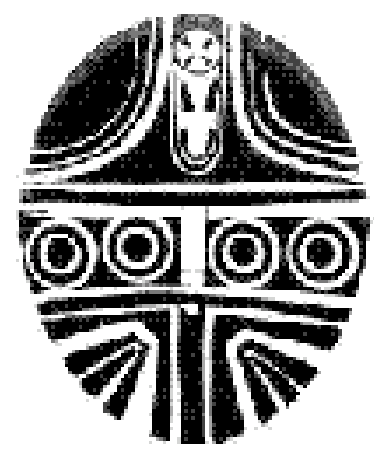

Fig. 16 
cordent alors avec leur disposition astronomique), soit en carré (le cas des poteaux centraux de la maloca), soit alignés (lorsqu'ils sont des "yeux ", et qu'il doivent de ce fait occuper cet espace de la face du masque sur lequel ils sont représentés). En cela, ces quatre "yeux" sont redevables du motif de la raie. Mais ce motif n'épuise pas le reste des dessins portés sur ces masques. Dans l'état actuel de la démonstration, nous ne pouvons pas encore expliquer les dessins qui prennent place sur les bajoues, les formes identiques mais inversées portées à hauteur de front, les lignes ve rticales et les cercles concentriques portés à cette même hauteur.

De même, si nous pouvons maintenant admettre que ces masques s'inspirent du motif de la raie, il n'est pas encore possible de les relier entièrement à la constellation d'Orion (où se trouverait, alors, les trois étoiles du Baudrier ?), ni d'y inclure les constatations astronomiques liées au parépou. Il nous faut, pour cela, introduire plusieurs données d'origine desana relevées par Reichel-Dolmatoff (1997: 266).

Le premier type de données évoque l'association des fruits de palmier avec Orion. Au moment des deux équinoxes, les Desana placent une grande quantité de fruits de palmier au centre de leur maloca, c'est-à-dire, pour eux, en correspondance avec $\varepsilon$ d'Orion. "En el centro, en épsilon Orión, en ciertas ocaciones rituales - en las dos fechas del equinoccio -, se depositan rimeros de frutas de palma". Bien que l'auteur n'indique pas de quel palmier il s'agit, nous pouvons raisonnablement penser que, coïncidant avec les équinoxes, les périodes de fructification du parépou permettent l'utilisation de ce fruit pour ces rituels.

Il est possible de déduire plusieurs implications de ces données. En premier lieu, les fruits de ce palmier sont associés au soleil équinoxial (ce que nous savions déjà). Deuxièmement, les fruits du même palmier sont associés aux trois étoiles du Baudrier d'Orion et plus particulièrement à epsilon $(\varepsilon)$ d'Orion. Nous devons ajouter qu'en plus du fait que $\varepsilon$ d' Orion est la plus brillante de ces trois étoiles, elle est également l'étoile centrale et du Baudrier et du Tr a pè ze de la constellation : son point de sortie à l'horizon oriental correspond - pour les Desana et d'autres sociétés amérindiennes - au point de sortie du soleil à l'équinoxe, ce qui expliquerait son association avec les fruits de parépou. Par rapport à la ligne d'horizon, il est donc possible d'affirmer que $\varepsilon$ d'Orion suit le même trajet dans le ciel nocturne que celui effectué par le soleil équinoxial dans le ciel diurne. D’un point de vue spatio-temporel, le soleil équinoxial est donc dans une relation de complémentarité et d’opposition avec $\varepsilon \mathrm{d}^{\text {'Orion }}{ }^{12}$. Nous pouvons en conclure qu'il existe une concordance spatio-temporelle qui fait que $\varepsilon$ d'Orion, le soleil à l'équinoxe et les fruits de palmier sont liés entre eux, et surtout que ce lien est reconnu par certaines sociétés amérindiennes de la région.

12. Claude Lévi-Strauss (1966: 239) avait déjà évoqué cette possibilité, sans toutefois la retenir par manque de données ethnographiques : «D’autre part, l'idée suggérée par les dernières lignes de $\mathrm{M}_{279 \mathrm{a}}$ [mythe des Kalina des Guyanes selon lequel "Orion appelle le soleil et le supporte"] qu' Orion pourrait être une contrepartie nocturne du soleil et son "support", pose le problème des phénomènes célestes, respectivement diurne et nocturne, mis en corrélation par la pensée indigène. Nous l'avons déjà re ncontré sur notre chemin et partiellement résolu dans un cas particulier : celui de l'arc-en-ciel et d'une zone obscure de la Voie lactée (CC, p. 252-53). Mais rien, actuellement, n’autorise à étendre le même raisonnement au soleil et à tout ou partie d'Orion». Nous pouvons donc admettre maintenant que cette correspondance existe entre le soleil équinoxial et $\varepsilon$ d'Orion. 


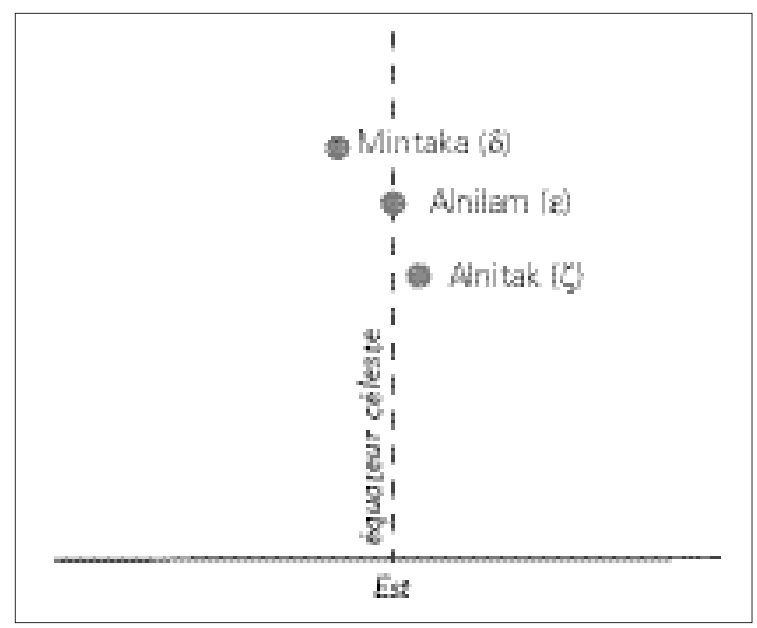

Fig. 18 Le Baudrier d'Orion, l'est et l'équateur céleste desana
Le second type de données prises aux Desana concerne un lien qu'ils instituent entre l'activité cérébrale - et plus généralement le cerveau humain - et le Baudrier d'Orion: "Las tres fases de tal proceso mental están representadas por Orión: el hemisferio cerebral izquierdo es Zeta Orión, el punto de transformación es épsilon, y el hemisfero derecho es Delta Orión" (ibid. : 268). Il peut apparaitre énigmatique que les Desana aient une concep-
pensée - ce qui laisserait suption si rationaliste du processus de latéralisation de la pensée - ce qui laisserait sup-
poser un artifice ethnographique de la part de Reichel-Dolmatoff. Mes prop res données d'ethno-anatomie miraña me permettent toutefois de confirmer qu'une telle possibilité ne relève pas d'une extrapolation de l'ethnographe ou de son informateur, tant l'architecture du cerveau humain, comme celle des primates à laquelle elle est facilement comparée, est connue et discutée aujourd'hui par les Miraña (Karadimas 1997). Quoi qu'il en soit, le fait d'associer ces trois parties de son architecture avec le Baudrier d' Orion ne peut, par contre, être une invention de l'ethnographe : elle relève d'un autre processus qui, justement, exploite des qualités de l'anatomie humaine pour les projeter - en pensée - sur đautres réalités avec lesquelles elle entretient un rapport de similarité structurale.

Les seules données miraña suffisent d'ailleurs à évoquer cette répartition des cercles sur les masques. Dans un rite de passage miraña à l'âge adulte tombé en désuétude par l'abandon de l'anthropophagie rituelle, les jeunes des deux sexes devaient recevoir une projection de quelques gouttes de sueur prélevées sur le visage d'adultes qui avaient mangé de la chair des ennemis : en l'occurrence de la moelle. Cette "sueur " était dénommée "la moelle de nos ennemis ", car elle était l'émanation de la moelle ingérée qui avait alors littéralement transformé l'essence des êtres qui l'avaient absorbée : elle devait faire resplendir la face des initiés. Les jeunes pouvaient alors, et seulement alors, connaître sexuellement un partenaire, et le visage des jeunes qui n'étaient plus vierges restait terne. Plusieurs adultes prenaient part au rituel qui se déroulait en début de soirée et se poursuivait toute la nuit. Chacun choisissait une localisation précise sur le visage des initiés. Il y avait cinq, voire six points sur le visage : on commençait par celui du milieu du front puis, de chaque côté de ce point, là où se trouve chacun des hémisphères cérébraux. Les pommettes recevaient chacune une goutte et, si les adultes qui projetaient cette sueur étaient nombreux, le menton pouvait parfois être le sixième et dernier point qui répondait symétriquement au premier sur le milieu du front. 
Selon les Miraña, le plus commun était de projeter sur les trois premiers points évoqués du visage - ceux du " haut»-, les trois autres étant le symétrique inverse des premiers - ceux du "bas» - selon un découpage horizontal du visage à partir d'une ligne imaginaire suivant une répartition « haut »/ «bas » formée par les sourcils, à la manière de l'iconographie des masques du rituel du parépou. Avant cela, les initiés devaient se nettoyer le visage avec le latex de trois arbres différents. En premier, un masque était confectionné en étalant le latex non cuit du même arbre, un ficus, qui sert à faire les masques pour les rituels du parépou (lors de la confection des masques du rituel ce latex est cuit, et forme la base noire en résine des "visages"), puis il était retiré et un second masque était confectionné en appliquant le latex de l'arbre à caoutchouc et enfin le latex de bábtsìtè (non-identifié) constituait le troisième masque qui était alors retiré après séchage. Suite à ces trois applications, le visage devait rendre de lui-même une graisse considérée comme "pure» par les Miraña. Au contact de la sueur, de la "moelle des ennemis ", la graisse des visages des initiés devenait luisante et resplendissante. Le visage devait alors "briller" comme un soleil dans la pénombre et les points de gouttes de sueurs avaient un éclat particulier : "comme celui d'une étoile". Avec l'éclat des deux yeux, les gouttes des pommettes formaient un trapèze, alors que le front devait briller en trois points.

Si nous combinons ces données issues de ces groupes, elles laissent entendre que $\varepsilon$ d'Orion occupe une place centrale dans le cerveau sur la scissure interhémisphérique cérébrale, les deux autres étoiles du Baudrier sur chacun des hémisphères, et l'ensemble est lié aux fruits de parépou (cette donnée prise aux Desana recoupe celle évoquée plus haut qui associe le racème de parépou et l'encéphale humain chez les Miraña). Enfin, $\varepsilon$ d'Orion et le soleil équinoxial étant interchangeables, il est également possible d'associer le soleil de ces périodes à la scissure interhémisphérique.

Un problème émerge cependant avec cette dernière donnée. En effet, si l'on prend la ligne dhorizon et l'équateur céleste comme lignes d'abscisse et d'ordonnée - sur l'équateur elles forment un angle droit -, le Baudrier d'Orion forme dans le ciel un alignement de trois étoiles de $45^{\circ}$ d'inclinaison, c'est-à-dire à mi-chemin entre la ve rticalité et l'horizontalité (comme elle est représentée sur une des poteries archéologiques du Nariño reproduite plus haut). Dans le cas où il s'agirait de figurer graphiquement le Baudrier d'Orion, trois possibilités d’ordre logique s'offrent au moins aux systèmes culturels : soit garder sa disposition oblique telle qu'elle apparaît dans le ciel, soit prendre le parti de lui faire subir une rotation de $45^{\circ}$ vers le haut, ou vers le bas (ve rticalité et horizontalité). Dans le cas d'une ro tation vers le haut, le Baudrier possède un alignement ve rtical qui suit l'équateur céleste. Il est alors associé au " chemin du soleil » (cf. infra). La seconde solution qui consiste à faire effectuer à l'alignement du Baudrier une rotation vers l'horizontalité crée une répartition symétrique qui permet de placer les trois étoiles suivant une latéralisation en opposition complémentaire avec leur centre : $\varepsilon$ d'Orion (ce dernier est parfois représenté décalé vers le haut pour respecter la forme de ligne brisée de l'alignement). De la sorte, chacune des étoiles occupe une place dans un hémisphère du ciel nocturne alors que $\varepsilon$ d'Orion reste sur la ligne de partage (l'équateur 
céleste). L'argument qui nous permettrait d'affirmer que les sociétés de la région optent plus pour ces deux solutions (alignement ve rtical et horizontal), plutôt que l'alignement à mi-chemin entre les deux $\left(45^{\circ}\right)$ est justement que les Desana répartissent ces trois étoiles suivant l'anatomie du cerveau ; ils se représentent donc leur environnement suivant une certaine idéologie corporelle. Associé au cerveau, il devient possible de donner à ces trois étoiles une place dans chaque hémisphère cérébral alors que $\varepsilon$ d'Orion occupe la scissure interhémisphérique.

Il devrait maintenant être possible d'associer ces constatations aux masques de la bière de parépou. Nous pouvons interpréter dans ce sens l'alignement des trois cercles sur la figure 8 formés chacun par plusieurs cercles concentriques qui se répartissent dans le haut du masque à la place que prendrait, sur une tête humaine, le cerveau avec les deux hémisphères et la scissure interhémisphérique. Dans le cas présent, $\varepsilon$ d'Orion y est représenté avec un diamètre supérieur à $\delta$ et $\zeta$ d'Orion puisqu'il les surpasse légèrement par sa magnitude (1,7 alors que $\zeta$ fait 1,8 et $\delta 2,2$ - l'échelle des magnitudes étant inversée; plus une étoile est brillante moins son indice est élevé).

Le motif de la figure 9 possède les mêmes trois cercles à hauteur de front avec ce qui devrait être $\varepsilon$ d'Orion marqué par un cercle plein et blanc. Sur la figure 13 , il semble que $\varepsilon$ d'Orion ait laissé place à une représentation de la scissure interhémisphérique alors que $\delta$ et $\zeta$ d'Orion sont représentés chacun dans un hémisphère cérébral. À l'inverse, sur la figure 16, il n’y aurait que $\varepsilon$ d'Orion qui est représenté dans cette scissure, alors que la figure 15 semble ne vouloir représenter que la scissure et l'architecture du cerveau vue de face, avec ses hémisphères représentés par des triangles dont la hauteur et l'hypoténuse sont courbes, suivant ainsi les contours des hémisphères cérébraux dans la boite crânienne. Les rayonnements qui en émanent semblent ainsi faire référence à l'autre valeur accordée à $\varepsilon$ d'Orion de pouvoir être interchangeable avec le soleil équinoxial.

Partant de ce dernier cas, les figures 10 et 11 devraient combiner les deux vues $-\varepsilon$ d'Orion et/ou soleil équinoxial - en substituant à la représentation de la scissure interhémisphérique de la figure 15 une représentation de l'étoile $\varepsilon$ d'Orion, mais dont la taille relative par rapport aux deux autres étoiles qui forment le Baudrier - et qui prennent, ici, la place des yeux - laisse à penser qu'elle rend également compte d'un soleil équinoxial. Avant d'aborder le motif d'un masque makuna qui évoque ce dernier cas, il nous faut revenir sur la représentation du Baudrier d' Orion associé aux yeux et à la scissure interhémisphérique (le motif miraña de la figure 7 rend parfaitement compte de cette modalité). Disposées de la sorte, les étoiles du Baudrier semblent évoquer «t rois yeux». Or les Urubu ou Kaapor de langue Tupi décrits par Francis Huxley (1980: 249) désignent le Baudrier d'Orion par cette même glose "les Trois Yeux ». Les motifs des masques de type $\mathrm{n}^{\circ} 7$ devraient donc représenter ce groupement de trois étoiles.

Placé de la sorte sur le milieu du front, $\varepsilon$ d'Orion est interchangeable avec le soleil à l'équinoxe. C'est en tout cas ce que laisse à penser le motif de la figure 19, que nous reproduisons ici et qui provient d'un masque d'un rituel makuna de la bière de parépou. 


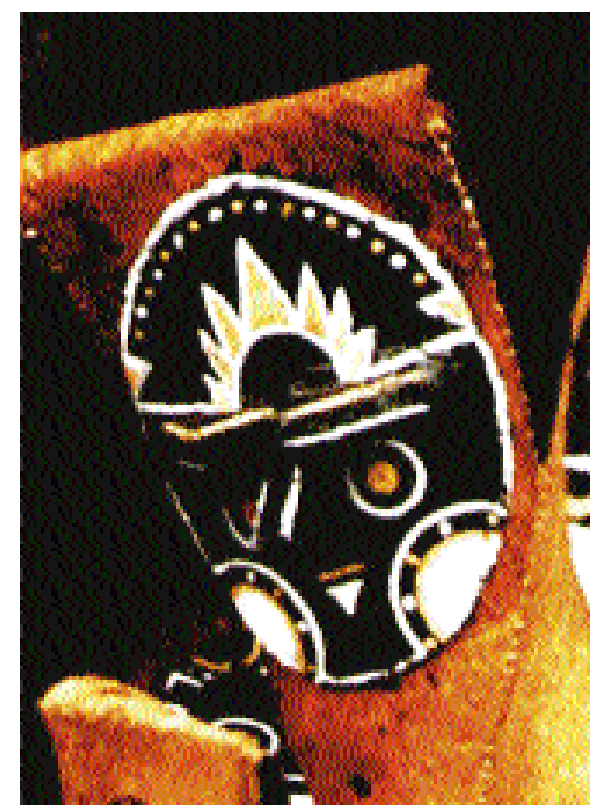

Fig. 19 Masque makuna (Århem 1998: 129)

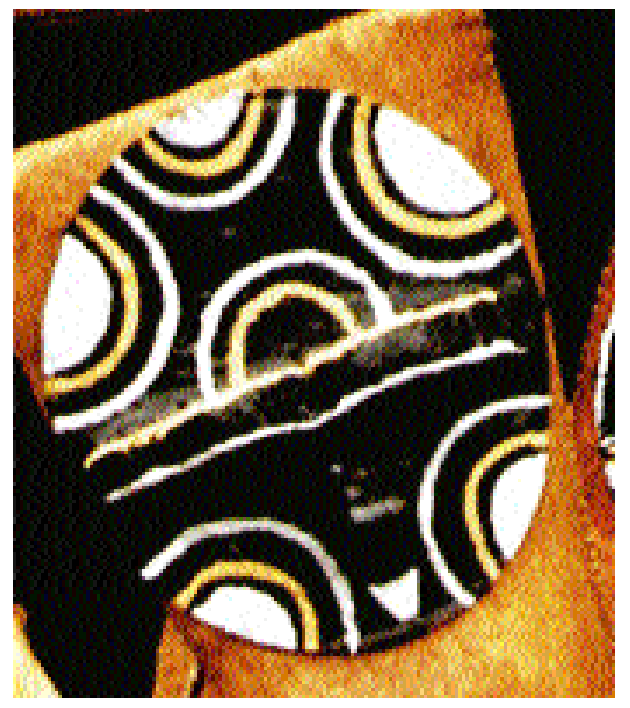

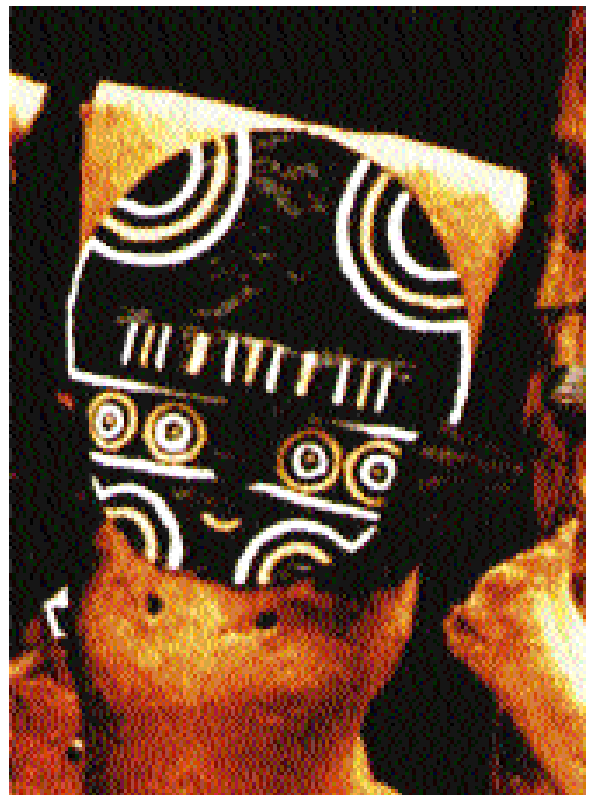

Fig. 20 Masque makuna (Århem 1998: 129)

Fig. 22 Masque makuna (Århem 1998: 129) 
Sur ce masque, il semble que le demi-cercle rayonnant placé au dessus de la ligne formée par les sourcils évoque un lever de soleil sur la ligne dhorizon et, dans la position centrale où il se trouve, d'un soleil équinoxial. La ligne horizontale formée sur les masques par des sourcils saillants représenterait alors la ligned horizon et le nez qui forme l'axe de symétrie, également saillant sur les masques, prendrait la place d'un gnomon. Tom Zuidema (1983 : 151) évoque une conception similaire chez les Incas, pour qui, et à certaines occasions rituelles, le nez était compris comme un gnomon, c'est-à-dire l'axe d'un cadran solaire :

«Describing various cult practices to the sun, it says that when people wanted to pray to the sun, they painted their nose yellow with a paste-like wax and that they painted their face. From data, which I analyse in other forthcoming articles (Zuidema ms.), we can conclude that the nose in this case symbolised a gnomon, measuring the passage of the sun through its zenith, and that as such it was an axis mundi.»

Nous devons faire une pause à ce stade de la démonstration et reprendre les éléments présents sur les masques et leurs interprétations astronomiques respectives. Les quatre "yeux " sont une référence au trapèze d'Orion, les alignements de trois cercles dans le haut des masques une référence au Baudrier et la ligne verticale et centrale qui les sépare, une évocation de l'équateur céleste sur lequel est centré $\varepsilon$ d'Orion. Vue de face, la ligne formée par les sourcils évoque l'horizon oriental et le nez un axe du monde.

Il nous faut maintenant expliquer les dessins qui apparaissent sur la plupart des masques à la place des bajoues et qui sont souvent reproduits de façon identique, mais renversée, sur le front. En effet, en association avec le Baudrier, ils peuvent être compris comme des évocations des hémisphères cérébraux, mais cette association n'est plus possible dès lors que le même motif est reproduit sur les bajoues. Le masque de la figure 20 donne un aperçu de ces motifs.

Nous savons, par l'analyse du mythe miraña, que ces masques sont une référence à un phénomène astronomique qui a lieu d'un solstice à l'autre et qui culmine avec l'équinoxe de septembre. Nous avons vu comment est représenté le soleil équinoxial, il manquerait donc des représentations des deux solstices.

C'est à nouveau une donnée d'origine desana qui va nous permettre de poursuivre la démonstration. Selon Reichel-Ddmatoff (1997 : 266), les Desana établissent la relation suivante entre les étoiles du trapèze d'Orion et les solstices: "... Betelgeuse y Bellatrix son los puntos del solsticio de verano, Saiph y Rigel los puntos del solsticio de invierno y al cinturón se le llama "Senda del Sol" "13.

Si les quatre "yeux " représentent les étoiles du trapèze d'Orion, ceux situés à gauche sur les masques devraient être Bellatrix $(\gamma)$ et Bételgeuse $(\alpha)$, et ceux de droite seraient Rigel $(\beta)$ et Saiph ( $\kappa)$ suivant leur répartition de part et d autre du nez (axe du monde et placé sur les masques dans la continuation de la scissure interhémisphérique).

Il faudrait alors considérer que les demi-cercles concentriques placés au bord du masque de la figure 20 sont liés à une paire « d'yeux » et qu'ils représenteraient

13. J'ai montré ailleurs que cette association est plus d’ord re calendaire que spatiale : le cycle d'Orion permet un découpage de l'année qui va d'un solstice à l'autre (Karadimas 1999). 
ainsi les levers et couchers du soleil aux deux horizons - oriental et occidental lors des deux solstices - juin pour ceux de gauche, et décembre pour ceux de droite. L'ensemble du masque serait ainsi une évocation de la totalité du ciel et le cercle extérieur qui délimite le "visage " utilisé comme une représentation de l'ensemble du cercle d'horizon. De celui-ci émergent des soleils (demi-cercles concentriques) placés latéralement par rapport à l'axe central (sortie équinoxiale du soleil). Il faut noter que, représentés de la sorte, ces masques reproduisent à peu de chose près une carte du ciel comme la ferait un astronome situé sur l'équateur s'il ne devait y inclure que la totalité de la ligne d'horizon en conjonction avec les levers et couchers solsticiaux et le trapèze d'Orion.

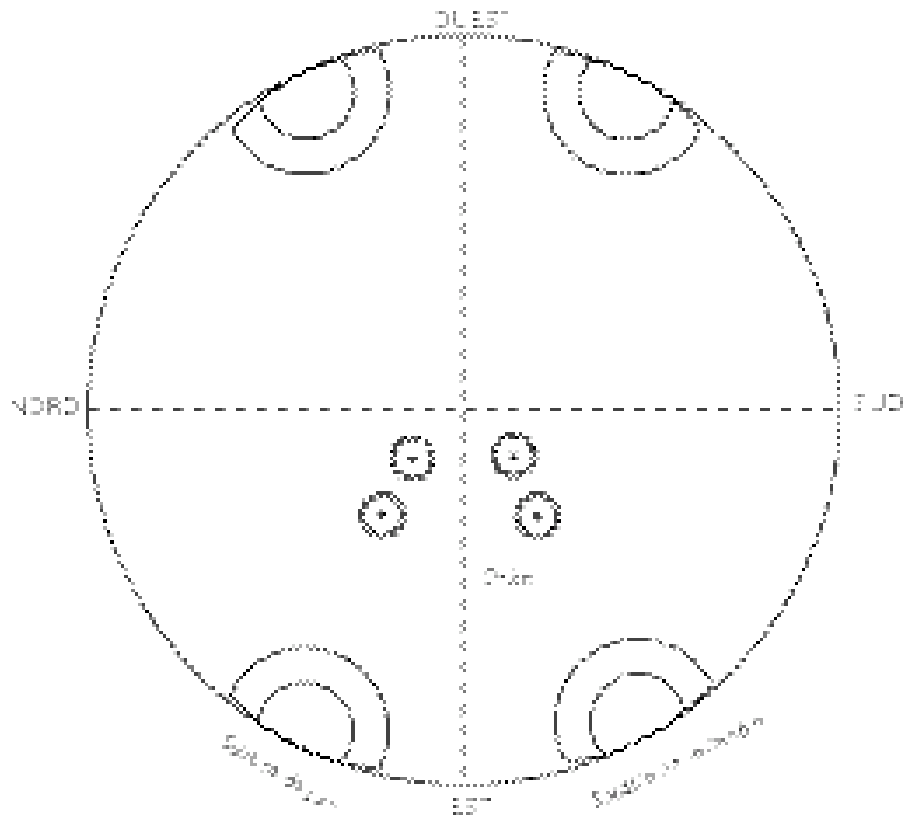

Fig. 2 I Vue globale du ciel, des quatre étoiles d'Orion et des solstices

Le motif du masque de la figure 22 combine ainsi les deux perspectives en apparence contradictoires d'une double représentation de l'horizon associé aux différentes phases solaires : la représentation de face du lever équinoxial oriental par la ligne formée par les sourcils dans le premier cas, et le cercle extérieur du visage associé aux solstices et augmenté, ici, de la position du coucher équinoxial (il est à noter que ce masque est alors dépourvu de cercles concentriques représentant les yeux).

Nous pouvons maintenant, grâce aux conceptions desana, réintroduire l'autre possibilité évoquée précédemment concernant les alternatives logiques s'offrant aux systèmes culturels de la région pour représenter le Baudrier d'Orion suivant une rotation verticale ou horizontale de son groupement d'étoiles. En alignant verticalement les trois étoiles du Baudrier, elles occupent la place de l'équateur céleste ou, dans le ciel diurne, le parcours solaire à l'équinoxe ("Chemin du 
Soleil»). Le masque miraña de la figure 6 possède, à la place de la scissure interhémisphérique, un alignement de deux triangles accolés symétriquement de part et d'autre d'un losange central. Dans ce cas, le Baudrier suit le «chemin du soleil» et $\varepsilon$ est figuré par une valeur entière - un losange - alors que $\delta$ et $\zeta$ d'Orion ne prennent, comme sur le motif du masque de la figure 10, que la moitié de cette valeur - c'est-à-dire des triangles - qui devrait évoquer les magnitudes relatives de ces étoiles entre elles.

L'utilisation des différentes figures mythiques - les quatre singes, la raie et Astre père et fils - par les groupes de la région permet des agencements qui trouvent leur accréditation dans la compréhension des phénomènes astronomiques liés aux cycles saisonniers. Le premier épisode du mythe rend compte d'une disposition particulière de l'architecture des maisons communautaires et figure sur des pièces archéologiques en provenance de la région des sources du Caquetá, alors que le second épisode trouve plus spécifiquement son illustration dans un rituel des groupes des basses terres baignées par ce même fleuve. Dans les deux cas, c'est le recours systématique à des parallèles entre certaines perceptions de l'environnement et leurs retranscriptions dans l'architecture et l'idéologie corporelle qui permet d'avoir accès au sens donné dans l'iconographie et dans les mythes. Par la conjonction entre trois identités successives - Raie, Anaconda des poissons et esprits -, les masques et surtout leurs motifs donnent à voir une sorte de définition par l'image de ce que sont les "êtres de l'ailleurs». Les esprits des animaux sont compris, dans le rituel, comme des visiteurs d'un autre monde, chtonien, où retournent se réfugier, de jour, les étoiles qui occupent le ciel nocturne. Venus, comme la raie, par l'est, ils "émergent " de jour d'un monde souterrain pour se rendre parmi les vivants.

Ces masques rendent ainsi compte d'une phase mythique associée à une conjonction de phénomènes astronomiques liés aux différents soleils solsticiaux qui "encadrent", aussi bien dans le temps que dans l'espace, le soleil de l'équinoxe de septembre. La seconde fructification du parépou se fait durant cet équinoxe alors que, paradoxalement, le rituel dans lequel sont présentés les masques se déroule autour de celui de mars. Mais ils sont en même temps plus que cela, puisque, par leurs motifs, ils donnent à voir une compréhension que peuvent avoir les sociétés amérindiennes de cette région de ces phénomènes astronomiques, mais aussi de leurs mythes et de leurs rituels dont ils ne font pas que reproduire la forme ou la succession des phases.

La compréhension des structures de l'image - que celle-ci soit un visage, des intérieurs de poteries ou des pièces d'orfevreries précolombiennes - permet d'avoir accès aux constructions intellectuelles, aux savoirs et aux systèmes de connaissance de ces sociétés. La simple reproduction des formes par les artisans indigènes lors de la confection des masques n'est pourtant pas le garant de l'inscription du sens en leur sein : ce qui permet à certains informateurs locaux de pouvoir affirmer que des masques réalisés par des jeunes sont «mal faits». Ces 
masques ne comportent alors plus trois ou quatre yeux qui permettraient de renvoyer à un référent connu par ceux qui perçoivent le sens du mythe et les motifs des masques, mais ils sont réduits à deux yeux comme en posséderaient les traits d'un simple visage humain.

Le niveau d'abstraction des représentations de ces masques qui, sous le couve rt des traits d'un visage ou d'un anthropomorphisme patent, font penser à une figuration complexe dêtres mythiques sans référence au réel, montre au contraire un extrême esprit de synthèse des différents savoirs liés à l'exécution de ce rituel. Si l'analyse des iconographies indigènes doit être tentée en termes anthropologiques, elle ne peut faire abstraction de l'assise mythique, du contexte rituel, comme des savoirs liés à la représentation du monde, au système catégoriel, mais surtout elle nécessite de mettre au jour les mécanismes mentaux ainsi que les outils mnémoniques utilisés par les systèmes culturels pour élaborer ces représentations.

MOTS CLÉSIKEYWORDS: Amazonie/Anazonia - Indiens miraña/Miraña Indian - ethnoastronomielethnoastronomy - masques/mask - rituel/ritual - mythologie/mythology.

\section{BIBLIOGRAPHIE}

Århem, Kaj

1998 Makuna: Portrait of an Amazonian People. Washington-London, Smithsonian Institution Press.

Baer, Gehard

1993 «Para o melhor entendimento das máscaras sul-americanas ", in Vera Penteado Coelho, ed., Karl von den Steinen: Um Século de Antropologia no Xingu. São Paulo, Universidade de São Paulo: 289-310.

\section{Burtch, Shirley}

1983 Diccionario Huitoto Murui.

Yarinacocha, Pucallpa (Perú), Instituto

Lingüístico de Verano, 2 vol.

Chaumeil, Jean-Pierre

2000-2001 «Le masque et le placenta: notes sur les costumes-masques yagua ", Bulletin de la Société suisse des Américanistes 64-65 : 97-105.

\section{Erikson, Philippe}

1996 «Le masque matis : matière à réflexion, réflexion sur la matière ", ms. (L'Homme, 2002, 161 : 149-164).
Goulard, Jean-Pierre

2000-2001 «Le costume-masque

(Tikuna) ", Bulletin de la Société suisse des Américanistes 64-65 : 75-82.

Guyot, Mireille

1975 "Le système cultural bora-miraña", in Pierre Centlivres, Jürg Gasche \& Alicia Lourteig, eds, Culture sur brûlis et évolution du milieu forestier en Amazonie du NordOuest, $\mathrm{n}^{\circ}$ spécial du Bulletin de la Société suisse d'ethnologie : 93-109.

Hartmann, Günther

1967 Masken sudamerikanischer Naturvölker. Berlin, Museums für Völkerkunde, Abteilung Amerikanische Naturvölker, I-13.

Huxley, Francis

1980 Aimables sauvages. Plon, Paris ("Terre

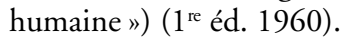

Karadimas, Dimitri

1997 Le Corps sauvage. Idéologie du corps et représentations de l'environnement chez les Miraña d'Amazonie colombienne. Nanterre, Université Paris X, thèse de doctorat. 
1999 «La constellation des Quatre Singes. Interprétation ethno-archéoastronomique des motifs de "El Carchi-Capulî"

(Colombie, Équateur) ", Journal de la

Société des Américanistes 85 (1) : 115-145.

2000-2001 «Singes, raies et étoiles entre les

Andes et l'Amazonie : perspectives comparatives dans l'iconographie amérindienne équatoriale ", Bulletin de la Société suisse des Américanistes 64-65 : 83-96.

Landaburu, Jon \& Roberto Pineda C.

1984 Tradiciones de la gente del hacha.

Mitología de los indios andoques del

Amazonas. Yerbabuena (Colombie), Caro y

Cuervo-Unesco.

\section{Lévi-Strauss, Claude}

1966 Mythologiques II. Du miel aux cendres. Paris, Plon.

1971 Mythologiques IV. L'Homme nu. Paris, Plon.

\section{Monod-Becquelin, Aurore}

1993 «O Homem Apresentado ou as Pinturas Corporais dos Índios Trumaís ", in Vera Penteado Coelho, ed., Karl von den Steinen: Um Século de Antropologia no Xingu. São Paulo, Universidade de São Paulo : 511-562.

Münzel, Mark

1971 Medizinmannwesen und

Geistervorstellungen bei den Kamayurá (Alto Xingú-Brasilien). Wiesbaden, Université de Francfort, thèse de doctorat.

\section{Musée d'ethnographie}

1975 Amazonie du Nord-Ouest. [Catalogue de l'exposition, 8 juin-31 déc.] Neuchâtel, Musée d'ethnographie-P. Attinger.

\section{Nimuendajú, Curt}

1952 The Tukuna. Berkeley-Los Angeles, University of California Press.

\section{Pãrõkumu, Umusî \& Tõrãmû Kêhíri}

1995 Antes o mundo não existia. Mitologia dos antiguos Desana-Kêhíripõrã. São Gabriel da Cachoeira, Amazonas (Brasil), União das nações indígenas do Rio Tiquié / Federação das organizaçôes indígenas do Rio Negro.

Reichel-Dolmatoff, Gérard

1973 Desana. Le symbolisme universel des Indiens Tukano du Vaupès. Paris, Gallimard.

1997 Chamanes de la Selva Pluvial. Ensayos sobre los Indios Tukano del Noroeste

Amazónico. Dartington, Green Books Ltd («Themis Books»).

\section{Van der Hammen, Maria Clara}

1992 El manejo del mundo. Naturaleza y sociedad entre los Yukuna de la Amazonía colombiana. Bogotá, Tropenbos Colombia.

\section{Vincent, William Murray}

1986 «Máscaras. Objetos rituais do Alto Rio Negro ", Suma etnolólogica brasileira. III : Arte india. Petrópolis, Vozes / Finep : 151-171.

\section{Whiffen, Thomas}

1915 The North-West Amazons. Notes of Some Months Spent among Cannibal Tribes. London, Constable \& Company.

\section{Zerries, Otto}

1961 «Die Tanzmasken der Tukuna- und Juri-Taboca- Indianer der Sammlung Spix und Martius im Staatlichen Museum für Völkerkunde zu München aus dem Jahre 1820 und ihre Bedeutung im Lichte neuer ethnologischer Forschung ", Paideuma 7 (7).

1981 «Kalebassenmasken und Idole bei ausserandien Indianern Sudamerikas ", Scripta Ethnologica (Buenos Aires) 6 : 173-185.

\section{Zuidema, Tom}

1983 " Masks in the Incaic Solstice and Equinoctial Rituals ", in M. Ross Crumrine \& Marjorie Halpin, eds, The Power of Symbols. Masks and Masquerade in the Americas. Vancouver, University of British Colombia Press : 150-163. 
Dimitri Karadimas, Le masque de la raie. Etude ethno-astronomique de l'iconog raphie d'un masque rituel miraña. — Lors d'un rituel équinoxial chez les Miraña d'Amazonie colombienne, les esprits des animaux sont invités à venir partager une boisson fermentée préparée à partir de fruits du palmier parépou. Lessentiel du rituel commémore une geste mythique dans laquelle les deux principaux protagonistes sont Soleil du Milieu et Raie (respectivement maître des animaux et maître des poissons). L'iconographie des masques qui représentent les différentes espèces animales est le plus souvent réaliste, sauf pour celui de Raie. En combinaison avec son assise mythologique, son iconographie prend un sens particulier lorsqu'elle est comparée à certaines conjonctions astronomiques de la constellation d' Orion dans le ciel équatorien. Les évocations spatiales et temporelles du rituel, du mythe et des singularités de l'environnement sont rendues par l'image des masques, qui devient un condensé de sens.
Dimitri Karadimas, Skate's Mask. An Ethnoastronomical Study of the Iconography of a Mask used in Miraña Ceremonies. - During an equinoctial ceremony among the Miraña in the Amazonian region of Colombia, animal spirits are invited to come share a fermented drink prepared with peach-palm nuts. The main part in this ceremony commemorates a mythical sequence with two major characters, Sun of the Middle and Skate Ray, the rulers of animals and fish respectively. The masks representing various animal species are usually realistic, except for Skate's. In combination with its basis in myths, this mask's iconography takes on special meaning when related to certain astronomical conjunctions of Orion near the equator. The spatial and temporal " evocations " of the ceremony, of the myth and of special features in the environment are rendered by the image of the masks, which condenses the meaning. 